\title{
The role of the bacterial protease Prc in the uropathogenesis of extraintestinal pathogenic Escherichia coli
}

Wen-Chun Huang ${ }^{1,2}$, Chung-Yen Lin ${ }^{3}$, Masayuki Hashimoto, 1,2, Jiunn-Jong Wu' ${ }^{5}$, Ming-Cheng Wang ${ }^{6}$, Wei-Hung Lin ${ }^{6}$, Chang-Shi Chen ${ }^{2,7}$ and Ching-Hao Teng ${ }^{1,2,4^{*}}$ (D)

\begin{abstract}
Background: Extraintestinal pathogenic E. coli (ExPEC) remains one of the most prevalent bacterial pathogens that cause extraintestinal infections, including neonatal meningitis, septicemia, and urinary tract (UT) infections (UTIs). Antibiotic therapy has been the conventional treatment for such infections, but its efficacy has decreased due to the emergence of antibiotic-resistant bacteria. Identification and characterization of bacterial factors that contribute to the severity of infection would facilitate the development of novel therapeutic strategies. The ExPEC periplasmic protease Prc contributes to the pathogen's ability to evade complement-mediated killing in the serum. Here, we further investigated the role of the Prc protease in ExPEC-induced UTIs and the underlying mechanism.
\end{abstract}

Methods: The uropathogenic role of Prc was determined in a mouse model of UTIs. Using global quantitative proteomic analyses, we revealed that the expression of FliC and other outer membrane-associated proteins was altered by Prc deficiency. Comparative transcriptome analyses identified that Prc deficiency affected expression of the flagellar regulon and genes that are regulated by five extracytoplasmic signaling systems.

Results: A mutant EXPEC with a prc deletion was attenuated in bladder and kidney colonization. Global quantitative proteomic analyses of the prc mutant and wild-type ExPEC strains revealed significantly reduced flagellum expression in the absence of Prc, consequently impairing bacterial motility. The prc deletion triggered downregulation of the flhDC operon encoding the master transcriptional regulator of flagellum biogenesis. Overexpressing fIhDC restored the prc mutant's motility and ability to colonize the UT, suggesting that the impaired motility is responsible for attenuated UT colonization of the mutant. Further comparative transcriptome analyses revealed that Prc deficiency activated the $\sigma^{E}$ and RcsCDB signaling pathways. These pathways were responsible for the diminished flhDC expression. Finally, the activation of the RcsCDB system was attributed to the intracellular accumulation of a known Prc substrate Spr in the prc mutant. Spr is a peptidoglycan hydrolase and its accumulation destabilizes the bacterial envelope.

Conclusions: We demonstrated for the first time that Prc is essential for full ExPEC virulence in UTIs. Our results collectively support the idea that Prc is essential for bacterial envelope integrity, thus explaining how Prc deficiency results in an attenuated ExPEC.

Keywords: Extraintestinal pathogenic Escherichia coli, Urinary tract infections, Protease Prc, Motility, Flagella, $\sigma^{E}$, Two-component signal transduction system RcsCDB, Spr

\footnotetext{
* Correspondence: chteng@mail.ncku.edu.tw

${ }^{1}$ Institute of Molecular Medicine, College of Medicine, National Cheng Kung

University, Tainan, Taiwan

${ }^{2}$ Institute of Basic Medical Sciences, College of Medicine, National Cheng

Kung University, Tainan, Taiwan

Full list of author information is available at the end of the article
}

(c) The Author(s). 2020 Open Access This article is distributed under the terms of the Creative Commons Attribution 4.0 International License (http://creativecommons.org/licenses/by/4.0/), which permits unrestricted use, distribution, and reproduction in any medium, provided you give appropriate credit to the original author(s) and the source, provide a link to the Creative Commons license, and indicate if changes were made. The Creative Commons Public Domain Dedication waiver (http://creativecommons.org/publicdomain/zero/1.0/) applies to the data made available in this article, unless otherwise stated. 


\section{Background}

Extraintestinal pathogenic Escherichia coli (ExPEC) is one of the most common bacterial pathogens causing bacteremia, neonatal meningitis, and urinary tract (UT) infections (UTIs) [1]. The diseases caused by ExPEC have resulted in substantial morbidity, mortality, and healthcare costs $[1,2]$. Antibiotic therapy is the traditional way to treat E. coli infections. However, the rapid emergence of antibiotic-resistant strains has become a serious problem in managing bacterial infections because of the shortage of novel and effective antibiotics [3]. Accordingly, new antimicrobial strategies against E. coli-associated infections are urgently needed. As bacterial factors required for maintaining the virulence of ExPEC are potential antimicrobial targets, identifying such factors and understanding how they contribute to infections would facilitate the development of novel treatment strategies.

The E. coli periplasmic protease Prc is required for ExPEC to cause a high level of bacteremia [4] since ExPEC lacking Prc displays enhanced sensitive to complement-mediated serum killing and thus is defective in survival in the host bloodstream [4]. In addition to that in ExPEC, Prc homologs in other pathogenic bacteria have also been shown to contribute to bacterial pathogenesis. For example, a prc mutant of Salmonella typhimurium exhibits a diminished ability to survive in murine macrophages and attenuated virulence in mice [5]. Disruption of the Prchomologous protein CtpA in the animal pathogens Brucella suis and Burkholderia mallei decreases the abilities of these bacteria to survive in murine macrophages [6,7]. Mutation of prc in the plant pathogen genus Xanthomonas results in decreased virulence, biofilm production, and resistance to environmental stresses $[8,9]$. In this study, we further demonstrate the novel pathogenic role of Prc in ExPEC UTIs.

The mechanism of how the Prc protease and its homologs contribute to bacterial virulence remains to be elucidated. Our previous study has shown that deletion of prc in the ExPEC strain RS218, which is associated with neonatal meningitis, significantly changed the protein profiles in the outer membrane (OM) fraction [4]. The altered protein expression in the $\mathrm{OM}$ fraction may contribute to the defective ability to cause infections since OM-associated proteins (OMPs) are the major factors involved in bacterium-host interactions and play key roles in maintaining the integrity of the OM, which is the main bacterial structure for sensing and coping with the harsh host environment during infections [10].

Flagella are the protein structures associated with the bacterial OM that mediate bacterial motility [11].
Flagella of ExPEC have been shown to contribute to the pathogenesis of UTIs because these structures enable the bacteria to disseminate, and they facilitate colonization and ascension of the UT [12-15]. The process of flagellum biogenesis is regulated by the flagellar regulon organized in a three-tier hierarchy [16]. Three flagellar genes, $f l h D, f l h C$, and $f l i A$, are central for the hierarchical expression of this regulon. At the top of this hierarchy (class 1) are the master operon genes, flhDC. Their gene products, FlhD and FlhC, assemble into a heterohexamer $\left(\mathrm{FlhD}_{4} \mathrm{C}_{2}\right)$ [17] that acts as an essential transcription activator of the class 2 genes. Class 2 genes encode the flagellum-specific sigma factor $\sigma^{28}$ (FliA), the flagellar basal body and hook proteins, etc. Class 3 genes encode the subunit of the flagellar filament (FliC), stator components of the flagellar motor, as well as the chemotaxis pathway.

The flagellar regulon is highly regulated by environmental cues, such as osmolality, nutrients, cell density, and temperature [18]. E. coli cells perceive and respond to such external environmental stimuli through extracytoplasmic stress signaling systems (ESSSs) whose activation triggers transcriptional reprogramming, allowing the bacteria to cope with the corresponding external conditions. Two-component signal transduction systems (2CSTSs) are among the members of ESSSs. The activation of some 2CSTSs, including RcsCDB, CpxA-CpxR, EnvZ-OmpR, and QseB-QseC, has been shown to suppress the expression of the flagellar regulon, [19-24]. In E. coli, the prototypical 2CSTSs consist of an inner membranebound sensor kinase and a DNA-binging cytoplasmic response regulator. In response to specific stimuli, the sensor kinase is autophosphorylated at a conserved histidine residue. Then, the phosphoryl group is transferred to a conserved aspartate in the cognate response regulator. Finally, the phosphorylated response regulator up- or downregulates the transcription of target genes to induce cellular responses to external signals. In addition to 2CSTSs, the alternative sigma factor $\sigma^{\mathrm{E}}$ governs an extracytoplasmic signaling pathway that responds to heat-shock stress [25, 26]. Although no study has demonstrated whether the activation of this heat-shock response system suppresses flagellum expression, it is known that bacterial motility is suppressed under high environmental temperatures, suggesting that the $\sigma^{\mathrm{E}}$ system may contribute to the regulation of the flagellar regulon [27]. In unstressed bacteria, $\sigma^{\mathrm{E}}$ is sequestered in the cytoplasmic side of the inner membrane by the antisigma factor RseA, which is an inner membrane-spanning protein with a periplasmic-exposed $\mathrm{C}$-terminus and a cytoplasmic-exposed N-terminus [28-30]. Under 
stress, RseA is proteolytically degraded by the sequential action of the periplasmic and cytoplasmic proteases DegS and RseP, resulting in release of $\sigma^{\mathrm{E}}$ into the cytoplasm, in which this sigma factor can associate with the core enzyme of RNA polymerase to allow $\sigma^{\mathrm{E}}$-regulated gene transcription [31-36]. The $E$. coli envelope, which is composed of the OM, inner membrane (IM), periplasm, and peptidoglycan mesh [37] is the frontline of bacterial interaction with the external environment. Alteration of the envelope components has been shown to be able to activate ESSSs, similar to environmental stresses. It remains unclear whether the altered protein profile of the OM fraction caused by Prc deficiency could activate the signaling systems.

As a periplasmic protease, Prc exerts its biological function through proteolytic regulation of its substrates, which are supposed to be located in or partially exposed to the periplasmic space. The attenuated virulence resulting from Prc deficiency in bacterial pathogens may be a consequence of the substrate dysregulation caused by the loss of proteolytic control. In E. coli, the periplasm-exposed OM protein Spr has been shown to be a substrate of the Prc protease. Spr is a peptidoglycan hydrolase. Singh et al. have shown that deletion of prc causes Spr accumulation in E. coli cells [38]. The protein accumulation contributes to the mutant's growth defect under low osmolarity at $42{ }^{\circ} \mathrm{C}$ [38].

In this study, we found that the Prc protease of ExPEC is necessary for maintaining intact bacterial motility that is important for UT colonization. The underlying mechanism was shown to be involved in the activation of ESSSs and the intracellular level of the Prc substrate Spr.

\section{Methods}

\section{Bacterial strains, plasmids and growth conditions}

The bacterial strains and plasmids used in this study are shown in Table 1. Bacteria were grown in Luria Bertani (LB) broth containing $1 \%$ tryptone, $0.5 \% \mathrm{NaCl}$ and $1 \%$ yeast extract at $37^{\circ} \mathrm{C}$ overnight (approximately $16 \mathrm{~h}$ ) and were stored in LB with a final concentration of $15 \%$ glycerol at $-80^{\circ} \mathrm{C}$.

\section{Construction of mutants and plasmids}

The ExPEC mutants were constructed using polymerase chain reaction (PCR) product-based $\lambda$ Red recombination, as described previously [42]. The plasmids pCA3 $\times$ Flag [43] and pKD3 [42] served as templates for synthesizing the $3 \times$ Flag- and chloramphenicol resistance cassette-encoding sequences by PCR, respectively. The primers used for mutant construction are shown in Additional file 1: Table S1.
The plasmids producing the Prc-S430A or Prc-K455A mutation proteins were generated by site-directed mutagenesis using the corresponding primer (Additional file 1: Table S1). The complementary and overexpression plasmids newly constructed in the study were created by cloning the indicated PCR-amplified gene fragments into the corresponding plasmid vectors. The primers for amplification of the gene fragments are shown at Additional file 1: Table S1.

\section{Mouse model of urinary tract infection (UTI)}

The animal UTI studies were performed as described previously [12], with some modification. For each experiment, 2 ExPEC strains were mixed at a ratio of 1:1. Eight-week-old female $\mathrm{C} 3 \mathrm{H} / \mathrm{HeN}$ mice were anesthetized and transurethrally inoculated with a $50-\mu$ l bacterial suspension $\left(1 \times 10^{8}\right.$ colony-forming unit, $\left.\mathrm{CFU}\right)$ per mouse using a sterile polyethylene catheter connected to an infusion pump (Harvard Apparatus, Holliston, MA, USA) with a flow rate of $100 \mu \mathrm{l} / \mathrm{min}$. Subsequently, $48 \mathrm{~h}$ post-infection, mice were sacrificed, and their bladders and kidneys were collected, weighed, and homogenized in sterile culture tubes containing $3 \mathrm{ml}$ of normal saline. Bacterial counts were differentiated and determined by plating the homogenates onto LB agar plates containing IPTG and X-gal. The strains with and without lacZ showed blue and white colonies on the plates, respectively.

\section{Liquid chromatography-tandem mass spectrometry (LC/ MS/MS) analysis of bacterial proteins}

To prepare bacterial proteins for LC/MS/MS analysis, 16-h cultures of WT-RS218 and $\Delta p r c-$ RS218 (three independent cultures for each strain) were harvested and subjected to French press at $8000 \mathrm{lb} . / \mathrm{in}^{2}$. The resulting bacterial lysates were subjected to $12.5 \%$ SDS-PAGE to separate the proteins in the samples. The gel lane of each sample was cut into 5 slices. The gel slices were subjected to in-gel digestion with trypsin, followed by protein identification with the Thermo LTQ-Orbitrap Velos system. The MS/MS spectra were searched against Escherichia coli SwissProt 2014_08 (546,238 sequences; 194,363,168 residues) using Sequest (version 27, rev 12), which is part of the BioWorks 3.3 data analysis package (Thermo Fisher, San Jose, CA, USA). Subsequently, protein identifications with 2 peptides in at least one of the samples were retained. The proteins that are defined to be located on or associated with the outer membrane (OM) based on the EcoCyc database (http://ecocyc.org/), showed at least a 2 -fold change with statistical significance between WT-RS218 and $\Delta p r c-$ RS218 were identified (Table 2). 
Table 1 E. coli strains and plasmids used in this study

\begin{tabular}{lll}
\hline Strains or & Relevant information & Reference \\
plasmids & \\
\hline Strains
\end{tabular}

Strains

WT-RS218

RS218 isolated from the cerebrospinal fluid of a neonate with meningitis

$\Delta$ prc-RS218

RS218 with a prc deletion

$\triangle$ lacZ-RS218

RS218 with a lacZ deletion

$\Delta$ lacZ $\Delta$ prc-RS218 RS218 with lacZ and prc deletions

$\Delta$ rseA-RS218 RS218 with a rseA deletion

$\Delta$ degS $\Delta$ prc-RS218 RS218 with degS and prc deletions

lacZ::

$\operatorname{deg} S \Delta \operatorname{deg} S \Delta$ prc-

RS218

$\Delta$ degS $\Delta$ prc-RS218 with complementary

degS at the lacZ gene chromosomal locus

$\Delta r c s B \Delta$ prc-RS218 RS218 with rcsB and prc deletions

lacZ::

rcsB $\triangle$ rcs $B \Delta p r c-$

$\triangle$ rcsB $\triangle$ prc-RS218 with complementary

RS218

$\Delta$ degS $\Delta r c s B \Delta p r c-\quad$ RS218 with $\operatorname{deg} S, r c s B$ and prc deletions RS218

$\triangle O m p R \Delta p r c-\quad$ RS218 with ompR and prc deletions

RS218

$\triangle q$ seBAprc-RS218 RS218 with qseB and prc deletions

$\triangle c p x R \Delta p r c-R S 218 \quad$ RS218 with cpxR and prc deletions

Spr-3xFlag-RS218 RS218 with spr-3xFlag tag at the spr gene chromosomal locus

Spr-3xFlag-Aprc- $\quad$ RS218 with spr-3xFlag tag and prc

RS218 deletion at the spr gene chromosomal locus

$\Delta$ spr $\Delta$ prc-RS218 RS218 with spr and pre deletions

$\Delta$ flic-RS218

RS218 with a fliC deletion

WT-CFT073

CFT073 isolated from the blood and urine of a woman with acute pyelonephritis

$\triangle$ prc-CFT073

CFT073 with a prc deletion

$\triangle$ lacZ-CFT073

CFT073 with a lacZ deletion

$\triangle \mathrm{ac} Z \mathrm{Aprc}$

CFT073

$\triangle$ degS $\Delta$ prc-

CFT073

$\triangle r c s B \Delta p r c-$

CFT073

$\triangle$ fliC-CFT073

CFT073 with lacZ and pre deletions

CFT073 with degS and prc deletions

CFT073 with rCsB and prc deletions

WT-UTI89

$\Delta$ prc-UTI89

$\triangle$ lacZ-UTI89

$\Delta$ lacZuprc-UT189

CFT073 with a flic deletion

UTI89 isolated from the urine of a patient with cystitis

$\Delta$ flic-UTI89

UT189 with a prc deletion

UTI89 with a lacZ deletion

UTI89 with lacZ and pre deletions

Plasmids

pCL1920

UTI89 with a fliC deletion

pPrc
$[4]$

[4]

This study

This study

This study

This study

This study

This study

This study

This study

This study

This study

This study

This study

This study

This study

This study

[39]

This study

This study

This study

This study

This study

This study

[40]

This study

This study

This study

This study
Table 1 E. coli strains and plasmids used in this study (Continued)

\begin{tabular}{lll}
\hline $\begin{array}{l}\text { Strains or } \\
\text { plasmids }\end{array}$ & Relevant information & Reference \\
\hline
\end{tabular}

pPrc-S430A

pCL1920 harboring the prc gene

containing a serine point mutation

This study resulting in inactivation of the serine protease

pPrc-K455A

PCL1920 harboring the prc gene containing a lysine point mutation resulting in inactivation of the serine protease

pUC19

pFlhDC

Expression plasmid containing the lac promoter

pUC19 harboring an HA-tagged flhD This study and a His-tagged flhC gene that are under control of the lac promoter

pACYC184

Expression plasmid containing constitutively expressed $\mathrm{Tet}^{\mathrm{r}}$ and

NEB

$\mathrm{Cm}^{r}$ genes

pDegQ

pACYC184 harboring the degQ gene that is under control of chloramphenicol resistant gene's promoter

pBAD

Expression plasmid containing an arabinose-inducible promoter

pBAD-FIhDC

pBAD harboring a HA-tagged flhD and a His-tagged flhC gene that are under control of an arabinose-inducible promoter

pRcsB

pBAD harboring the $\operatorname{rCs} B$ gene that is under control of an arabinose-inducible promoter

pRseA

pBAD harboring the HA-tagged-rseA gene-His-tagged that is under control

This study

NEB of an arabinose-inducible promoter

pSpr

pBAD harboring a Flag-tagged spr gene that is under control of an arabinose-inducible promoter

\section{Western blot analysis}

The protein levels in the OM fractions or bacterial lysates were determined by western blot analyses. Preparation of the protein samples was performed as described previously [4]. The primary antibodies utilized to detect FliC, Prc, and OmpA were rabbit antisera against FliC (anti-H7, Becton Dickinson, Sparks, MD, USA) and Prc, and a mouse anti-OmpA antiserum. The recombinant proteins fused with HA and Flag tags were detected with a mouse anti-HA antibody and rabbit anti-Flag antibody, respectively (Sigma-Aldrich, St. Louis, MO, USA).

\section{Motility assay}

Bacterial strains were stab inoculated onto $0.3 \%$ agar plates and incubated at $37^{\circ} \mathrm{C}$ for $10 \mathrm{~h}$ [44]. The diameter of motility was measured and is shown in the quantified figure. 
Table 2 Identification of altered OMPs by liquid chromatography-tandem mass spectrometry

\begin{tabular}{|c|c|c|c|}
\hline Protein name & Description & Fold change ${ }^{a}$ & $P$ value \\
\hline \multicolumn{4}{|c|}{ Downregulated protein in $\Delta$ prc-RS218 } \\
\hline FliC & Flagellin & $(-) 100$ & 2.50E-04 \\
\hline SlyB & Outer membrane lipoprotein SlyB & $(-) 100$ & 5.30E-04 \\
\hline \multicolumn{4}{|c|}{ Upregulated protein in $\Delta p r c-R S 218$} \\
\hline Spr & Murein DD-endopeptidase & (+) 100 & $1.10 \mathrm{E}-03$ \\
\hline BamA & Outer membrane protein assembly factor & (+) 100 & 3.30E-04 \\
\hline Ag43 & Antigen 43 & (+) 100 & 7.80E-04 \\
\hline Tsx & Nucleoside-specific channel-forming protein & (+) 100 & $9.78 \mathrm{E}-07$ \\
\hline TraT & Conjugal transfer surface exclusion protein TraT & (+) 3.17 & $1.55 E-02$ \\
\hline TolC & Outer membrane channel protein TolC & (+) 2.21 & 4.47E-03 \\
\hline
\end{tabular}

${ }^{\mathrm{a}}(-)$, indicates that the protein was downregulated in $\Delta p r c-\mathrm{RS} 218$ compared to in WT-RS218

$(+)$, indicates that the protein was upregulated in $\Delta$ prc-RS218 compared to in WT-RS218

\section{RNA isolation}

Total RNA was extracted from 16-h cultures of bacteria using the RNeasy Mini kit (QIAGEN, Hilden, Germany) according to the manufacturer's instructions. To remove contaminating DNA, the resulting RNA was incubated with DNase I (Roche Applied Science, Mannheim, Germany) at $37^{\circ} \mathrm{C}$ for $1.5 \mathrm{~h}$. Then, the mixture was subjected to phenol / chloroform (1:1) (SigmaAldrich, St. Louis, MO, USA) extraction and ethanol precipitation. Finally, the purified RNA was dissolved in RNase-free water and stored at $-80{ }^{\circ} \mathrm{C}$.

\section{RNA sequencing (RNA-seq) and identification of differentially expressed genes}

The total RNA samples from the WT-RS218 and $\Delta p r c-$ RS218 16-h cultures (three independent culture samples for each strain) were subjected to cDNA library construction (paired-end) using a TruSeq RNA sample preparation kit (Illumina, San Diego, CA, USA). Sequencing was performed with the Illumina Solexa platform according to the manufacturer's protocol (Illumina, San Diego, CA, USA). Qualified reads were aligned to the reference genome of the ExPEC strain UTI89 because the genomes of RS218 and UTI89 are very similar [40, 45]. The transcriptional level of gene expression was calculated and normalized by fragments per kilobase of transcript per million mapped reads (FPKM) [46]. The differentially expressed genes between the wild-type strain and the prc mutant ( $P$ value $<0.05$ ) were analyzed by Regulatory Network Interactions of RegulonDB database [47] and classified into different regulons (Table 3 ).

\section{Real-time quantitative PCR (qPCR) and reverse} transcription-PCR (RT-PCR)

The purified RNA was reverse transcribed into cDNA by using random hexamer primers and Moloney murine leukemia virus (M-MLV) reverse transcriptase according to the manufacturer's instructions (Invitrogen, Carlsbad, CA, USA). For qPCR, the cDNA and primers were mixed with KAPA SYBR FAST qPCR Master Mix (Kapa Biosystems, Boston, MA, USA) and then subjected to PCR using a StepOnePlus ${ }^{\mathrm{Tm}}$ RealTime PCR System (Applied Biosystems, Carlsbad, CA, USA). The expression levels of the genes were normalized to those of $f t s Z$. For RT-PCR, the cDNA was subjected to PCR amplification using Taq polymerase for 25 cycles. The resulting products were analyzed by gel electrophoresis and visualized by ethidium bromide $(\mathrm{EtBr})$ staining. The primers used for these assays are shown in Additional file 1: Table S1.

\section{Statistical analysis}

Animal UTI experiments were analyzed by using a nonparametric Wilcoxon matched-pair test. The statistical significance of the other experiments were analyzed by unpaired two-tailed Student's $t$ test. A $P$ value of $<0.05$ was considered statistically significant. *, $P<0.05 ;$ ** $P<0.01 ;$ **; $P<0.001$; and ****, $P<$ 0.0001. (GraphPad Prism 7; GraphPad Software Inc., La Jolla, CA, USA).

\section{Results}

Prc deficiency decreases the ability of extraintestinal pathogenic Escherichia coli (ExPEC) to cause urinary tract (UT) infections (UTIs)

We have previously shown that Prc is required for ExPEC to cause a high level of bacteremia [4]. Since ExPEC is the most common cause of UTIs, in the present study, we further investigated whether Prc contributes to ExPEC UTIs. The ability of a prc mutant of the ExPEC strain RS218 ( $\Delta p r c-R S 218$ ) to colonize the UT was compared to that of an otherwise wild-type lac $Z$ mutant ( $\Delta$ lacZ-RS218). Deletion of lacZ did not affect 
Table 3 The differentially expressed flagellum- and five ESSSSrelated genes between WT-RS218 and $\Delta$ prc-RS218 by RNA-seq analysis

\begin{tabular}{|c|c|c|}
\hline $\begin{array}{l}\text { Gene } \\
\text { name }\end{array}$ & Gene product / functional description & $\begin{array}{l}\text { Fold } \\
\text { change }^{\text {a }}\end{array}$ \\
\hline
\end{tabular}

Flagellum-related genes

flhD Transcriptional activator of flagellar $\quad 0.73$ class II operons

$0.73 \quad 0.019$

fliT Chaperone of flagellar export system

flip Flagellin export apparatus, integral membrane protein

fliE $\quad$ Flagellar synthesis; basal body component

fliD Hook-associated protein 2

0.31

$\sigma^{\mathrm{E}}$ regulon

wza

wzC

hpf

uspD

rpoN

yiis

iaaA

Polysaccharide export protein

Tyrosine-protein kinase Etk/Wzc

9.12

3.49

2.66

2.50

2.46

2.42

2.40

ecfJ

\section{year}

insk

Universal stress protein $D$

RNA polymerase subunit, $\sigma^{N}$

Unknown function

Beta-aspartyl-peptidase

Unknown function

Predicted lipoprotein

IS150 conserved protein Insb, integrase core domain protein

fabz 3R-hydroxymyristoyl acyl carrier protein (ACP) dehydratase

bamE Lipoprotein stabilizer of BamABCDE OM biogenesis complex

\section{rseA Anti-RNA polymerase sigma factor $\sigma^{E}$}

tolB Tol-Pal cell envelope complex

clpX ATPase subunit of ClpXP protease

rutR TetR/AcrR family transcriptional regulator

mdoG Periplasmic glucan biosynthesis

IptD LPS assembly outer membrane complex protein

plsB Glycerol-3-phosphate O-acyltransferase

rfaD ADP-L-glycero-D-mannoheptose-6epimerase

fkpA Periplasmic peptidylprolyl cis-trans isomerase

Rcs $B$ regulon

$\begin{array}{ll}\text { yjbE } & \text { Exopolysaccharide production protein } \\ \text { wza } & \text { Polysaccharide export protein } \\ \text { wzC } & \text { Tyrosine-protein kinase Etk/WzC } \\ \text { osmC } & \text { Osmotically inducible protein OsmC } \\ \text { osmB } & \text { Osmotically inducible lipoprotein } \\ \text { rarA } & \text { Recombinase RarA } \\ \text { CpxR regulon } \\ \text { mviM } \quad \text { Putative virulence factor }\end{array}$

Table 3 The differentially expressed flagellum- and five ESSSsrelated genes between WT-RS218 and $\Delta$ prc-RS218 by RNA-seq analysis (Continued)

\begin{tabular}{|c|c|c|c|}
\hline $\begin{array}{l}\text { Gene } \\
\text { name }\end{array}$ & Gene product / functional description & $\begin{array}{l}\text { Fold } \\
\text { change }^{a}\end{array}$ & $\begin{array}{l}P \\
\text { value }\end{array}$ \\
\hline aroG & $\begin{array}{l}\text { 2-dehydro-3-deoxyphosphoheptonate } \\
\text { aldolase }\end{array}$ & 1.93 & 0.004 \\
\hline$m d t C$ & MdtABC-TolC efflux pump & 1.52 & 0.026 \\
\hline slt & Soluble lytic murein transglycosylase & 1.49 & 0.02 \\
\hline ppiA & Peptidylprolyl cis-trans isomerase A & 1.16 & 0.012 \\
\hline$m d t B$ & MdtABC-TolC efflux pump & 1.15 & 0.002 \\
\hline \multicolumn{4}{|c|}{ QseB regulon } \\
\hline qseB & $\begin{array}{l}\text { Quorum sensing two-component } \\
\text { response regulator }\end{array}$ & 1.50 & 0.018 \\
\hline \multicolumn{4}{|c|}{ OmpR regulon } \\
\hline ompC & Outer membrane porin protein $C$ & 3.29 & 0.031 \\
\hline
\end{tabular}

the ability of ExPEC to colonize the UT (data not shown). After transurethrally infecting a 1:1 mixture of the two bacteria into mice for $48 \mathrm{~h}$, the bacterial loads in the bladder and kidney were determined. $\Delta p r c-$ RS218 showed significantly lower bacterial counts than the otherwise wild-type strain in the bladder and kidney (Fig. 1a). In addition, transcomplementation with the prc gene significantly restored the ability to colonize the organs (Fig. 1b). Similarly, prc mutants of the ExPEC strains CFT073 and UTI89, which were isolated from patients with UTIs, showed decreased abilities to colonize the UT, and trans-complementation of the mutants with the prc gene restored their bacterial counts in the organs (Fig. 1c, d, e, and f). These results demonstrated that Prc contributes to the pathogenesis of UTIs.

\section{Prc deficiency alters the expression of outer membrane (OM)-associated proteins (OMPs) in ExPEC}

It has been demonstrated that deletion of prc alters the OMP profile in RS218 [4]. As shown in Fig. 2, prc deletion changed the OMP profiles of CFT073 and UTI89 as well. These findings suggested that alteration of OMP expression is a common outcome of Prc deficiency in ExPEC strains.

The OMPs of pathogenic bacteria are often involved in the host-bacterium interaction during the course of infections [48]. This fact led us to speculate that the altered OMP expression in the $p r c^{-}$ExPEC strains may be responsible for the defect in UT colonization. To determine the OMPs differentially expressed in ExPEC with and without $p r c$, the proteomic profiles of WT-RS218 and $\Delta p r c$-RS218 were determined by liquid chromatography-tandem mass spectrometry (LC/ MS/MS). The levels of 25 and 32 proteins were 


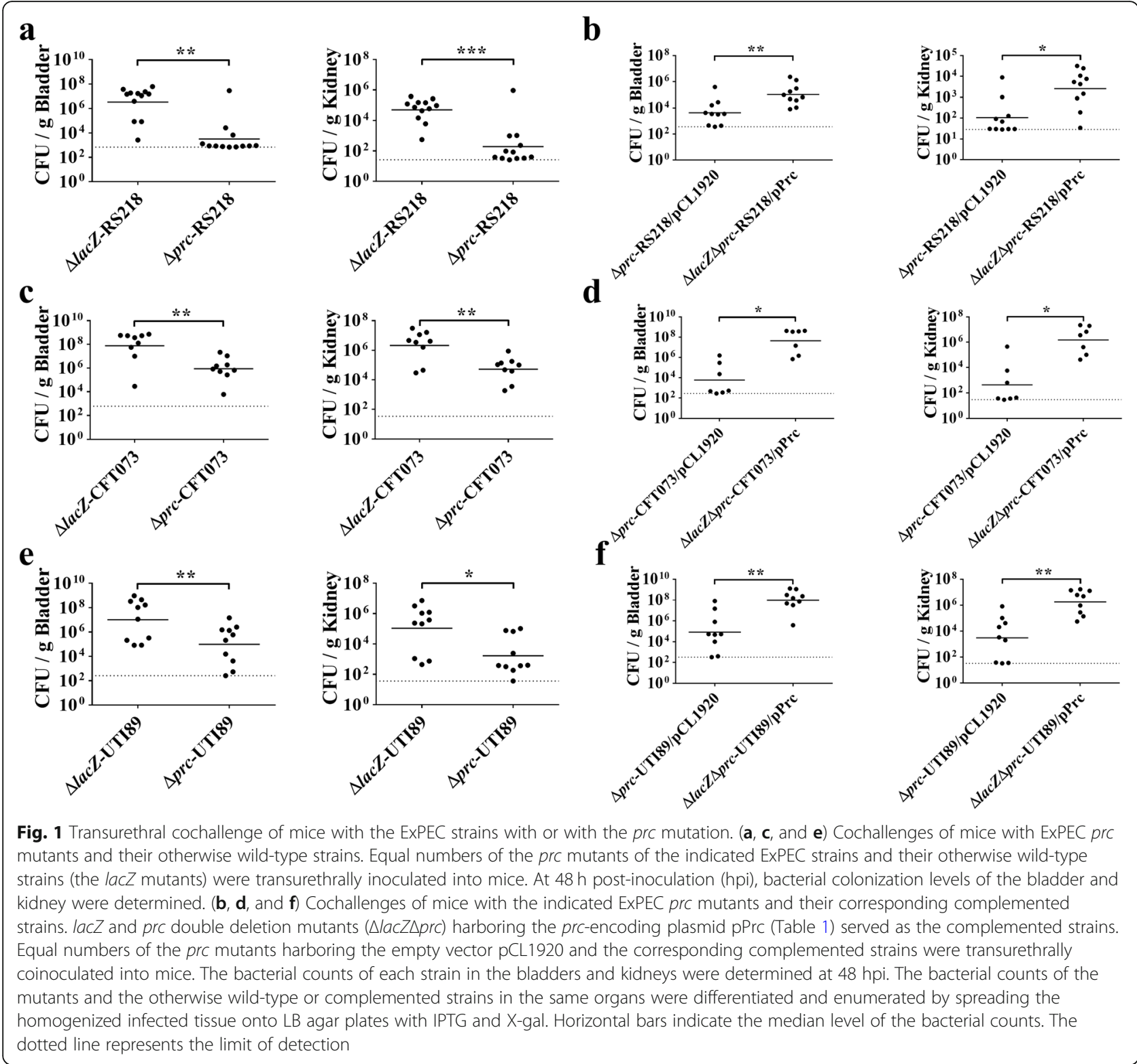

shown to be significantly downregulated and upregulated, respectively, by the prc deletion (Additional file 2: Table S2). Among them, 2 OMPs (FliC and SlyB) were upregulated in $\Delta p r c$-RS218, while 6 OMPs (Spr, BamA, Tsx, TolC, Ag43 and TraT) were downregulated in the prc mutant (Table 2).

\section{Prc deficiency decreases the flagellin level and motility of EXPEC}

While identified in the LC/MS/MS analysis, FliC (flagellin) is the major component of the flagellar filament, and flagellum-mediated motility is required for ExPEC to cause UTIs [12-15]. Thus, our study subsequently focused on the FliC protein.
To confirm the LC/MS/MS results regarding FliC, the levels of FliC in WT-RS218 and $\Delta p r c-$ RS218 were further determined by western blot analysis with an antiFliC antiserum. Consistently, $\Delta p r c-$ RS218 showed lower levels of FliC in total bacterial lysate and the OM fraction than WT-RS218 (Fig. 3a left panel). In addition, the $p r c$ mutant showed a significantly lower motility than the wild-type strain (Fig. 3a right panel). Trans-complementation of $\Delta p r c$-RS218 with the prc gene restored the expression of FliC and motility to the levels of those of WT-RS218. Similar phenotypes were also shown in CFT073 and UTI89 (Fig. 3b and b). These results demonstrate that Prc deficiency reduces flagellin (FliC) expression and suppresses bacterial motility in ExPEC. 


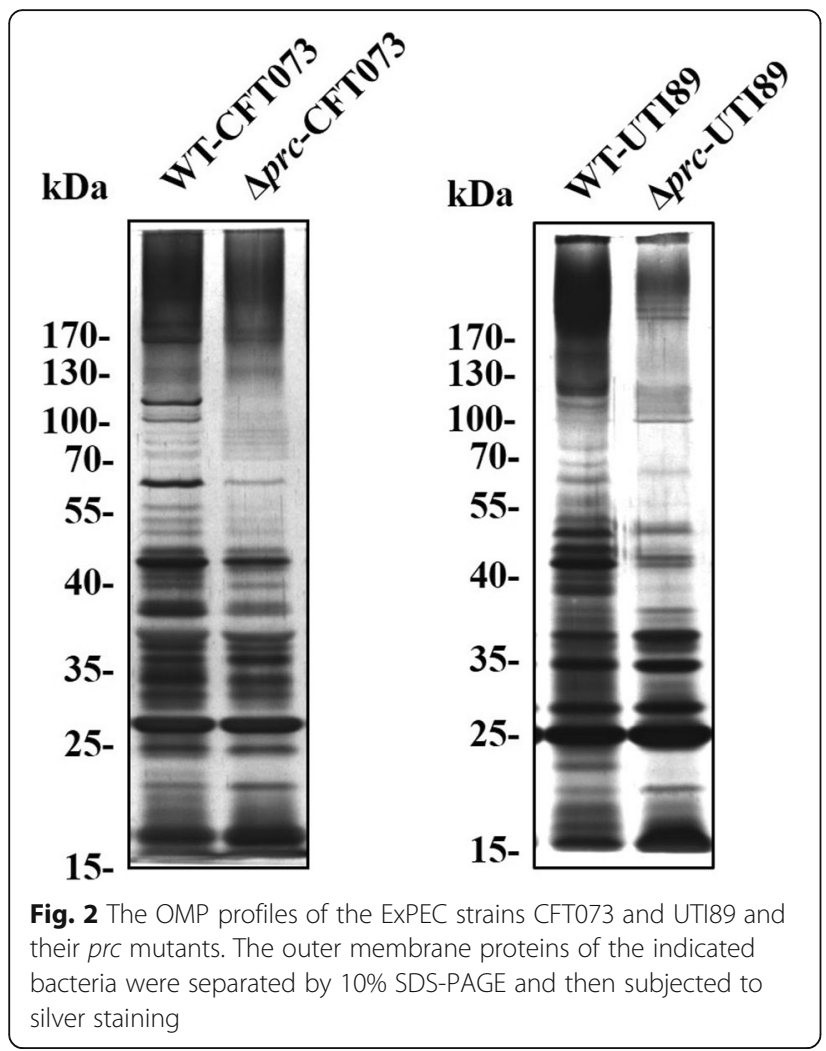

Prc deficiency decreases the expression of the flagellar regulon

$\mathrm{fliC}$ is located at the lowest level (class 3) of the transcriptional hierarchy of the flagellar regulon [16]. We investigated whether Prc deficiency affects the expression of this regulatory cascade. $\Delta$ prc-RS218 exhibited lower expression levels of the class 1 (flhD), class 2 (fliE, fliF, flhA, flgE, flgM, fliM, fliT, and fliA) and class 3 (fliC and motA) genes than WT-RS218 (Fig. 4). Trans-complementation of $\Delta p r c-\mathrm{RS} 218$ with prc restored the expression of these genes, suggesting that Prc deficiency results in the downregulation of all classes of genes in the regulon. Given that the top master operon $f l h D C$ governs all genes in this regulon, it is likely that prc deletion suppresses $f l h D C$ expression to cause the reduced motility. To assess this speculation, we examined the motilities of $\Delta p r c$-RS218 strains with different levels of $f l h D C$ expression. To do so, $\Delta p r c$ RS218 was transformed with a $f l h D C$-containing plasmid, pBAD-FlhDC (Table 1). Because the flhDC operon in this plasmid was driven by an arabinose-inducible promoter, arabinose treatment dose-dependently induced the expression of FlhDC (Fig. 5a). The induction of $f l h D C$ expression also dose-dependently increased the FliC level and bacterial motility (Fig. 5a and b). These results supported that through suppressing the expression of $f l h D C$, prc deletion downregulates the whole flagellar regulon and consequently decreases bacterial motility.

\section{The defective motility is responsible for the decreased} ability of the prc mutant to cause UTIs

To further investigate whether the defective motility caused by prc deletion contributes to the attenuated ability to cause UTIs, we promoted the motility of prc $^{-}$ ExPEC strains and then assessed their abilities to cause UTIs. The plasmid pFlhDC, which can constitutively overexpress $f l h D C$, was introduced into the $\mathrm{prc}^{-}$strains of RS218, CFT073, and UTI89 and was able to increase their motilities (Fig. 6a). The pFlhDC-harboring prc mutants showed higher levels colonization than the corresponding empty vector-harboring strains (Fig. 6b, $c$, and d). These results demonstrated that the decreased motility is responsible for the defective ability of prc mutants to colonize the UT.

\section{Deficiency in Prc increases the expression of the genes governed by some ESSSs}

To investigate how Prc deficiency results in the downregulation of $f l h D C$ transcription, comparative transcriptome analysis of $\Delta p r c-$ RS218 and WT-RS218 by RNAseq was performed. The prc deletion significantly affected the transcriptome of ExPEC, in which 152 and 365 genes were upregulated and downregulated, respectively (Additional file 3: Table S3). Consistently, many genes in the flagellar regulon were shown to be significantly downregulated in the prc mutant (Table 3). In addition, genes known to be able to be upregulated by some extracytoplasmic stress signaling systems (ESSSs), including the $\sigma^{\mathrm{E}}$, RcsCDB, CpxA-CpxR, QseB-QseC, and EnvZ-OmpR systems, showed significantly higher expression levels in $\Delta p r c-\mathrm{RS} 218$ than in WT-RS218 (Table $3)$. The differential transcript levels of the representative genes governed by these ESSSs were further confirmed by RT-PCR analysis (Fig. 7). These findings suggest that these ESSSs may be activated in the prc mutant. It is likely that the activated ESSSs contribute to the reduced motility.

\section{Blocking the activation of the $\sigma^{\mathrm{E}}$ or RcsCDB system} partially restores the motility of the prc mutant of ExPEC If the ESSSs play roles in suppressing the motility of the prc mutant, blocking their activation may relieve the suppression. As deletion of the $\operatorname{deg} S, \operatorname{rcs} B, c p x R, q s e B$, and $o m p R$ genes blocks the activation of the $\sigma^{\mathrm{E}}$, RcsCDB, CpxA-CpxR, QseB-QseC, and EnvZ-OmpR systems [35, $49,50,56,57]$, respectively, the deletion of these genes was introduced into $\Delta p r c-\mathrm{RS} 218$ to inactivate the corresponding systems. As shown in Fig. 8a, the $\operatorname{degS}$ and rcs $B$ deletions partially restored the motility of $\Delta$ prcRS218, while the $c p x R, q s e B$, and $o m p R$ deletions showed 


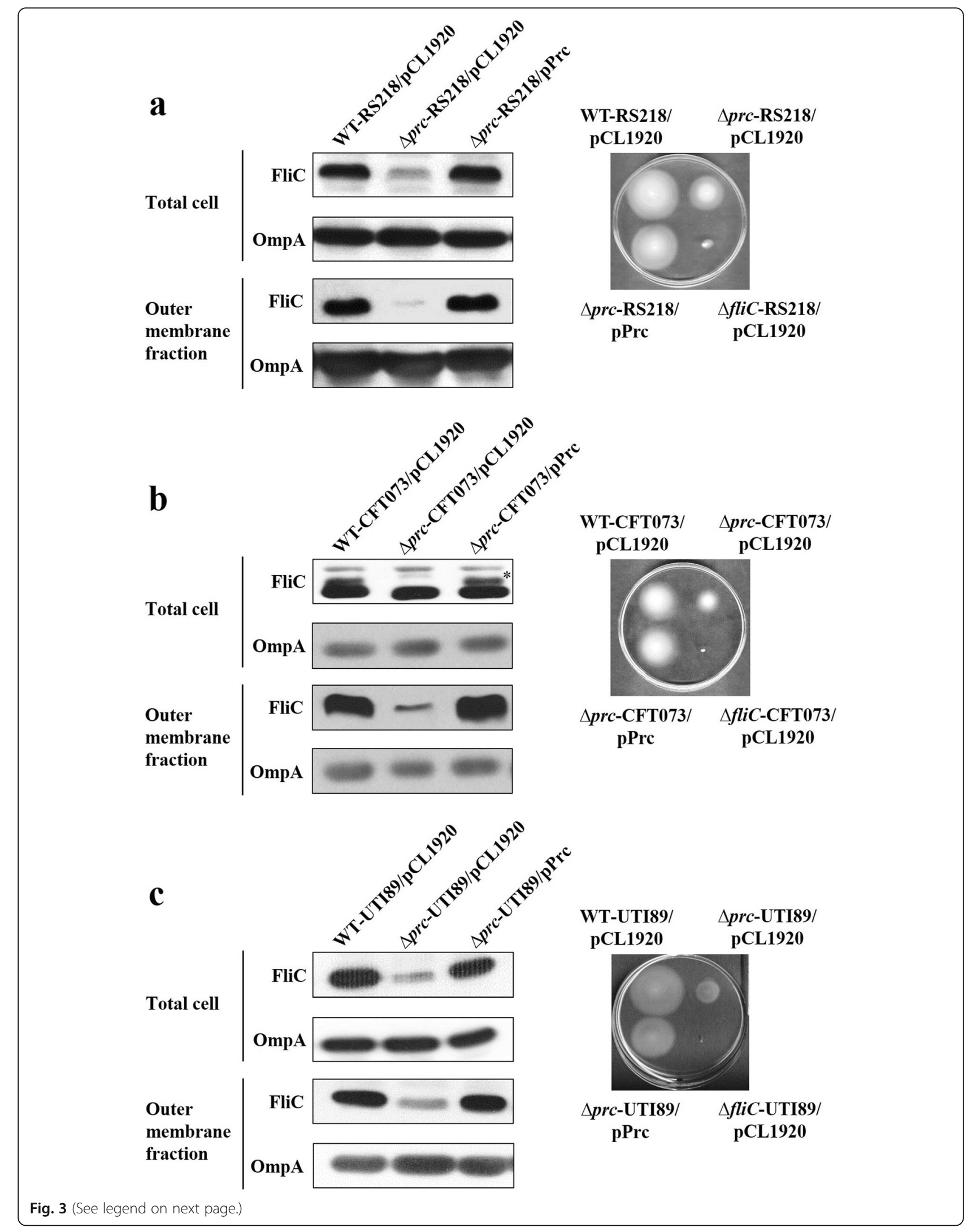


(See figure on previous page.)

Fig. 3 The levels of FliC expression and motility of RS218, CFT073, UTI89, and their prc mutants. The effects of prc deletion on FliC expression and motility in the ExPEC strains RS218 (a), CFT073 (b), and UTI89 (c). The results of western blot analyses of the total cell lysates and the OM fractions of the indicated bacterial strains are shown in the left panels of (a), (b), and (c). OmpA served as a loading control. The motilities of the indicated strains on $0.3 \%$ agar plates are shown in the right panels of (a), (b), and (c). pCL1920, the empty plasmid vector; pPrc, the plasmid pCL1920 harboring prc (Table 1). The asterisk indicates the FliC protein in the total lysate of CFT073

no significant effect on bacterial motility. Complementation of the double mutant strains $\Delta$ degs $\Delta p r c$-RS218 and $\Delta r c s B \Delta r c$-RS218 with $\operatorname{degS}$ and $r c s B$ in the chromosomal lacZ locus, respectively, decreased the motilities of these strains to the level of that of the prc single mutant (Fig. 8b and c), suggesting that activation of the $\sigma^{\mathrm{E}}$ and RcsCDB systems is involved in the decreased motility of the prc mutant. Similar results were also shown in CFT073 (Fig. 8d and e).

The activated $\sigma^{\mathrm{E}}$ and RcsCDB systems are involved in the suppression of FliC expression and flhDC transcription in $\Delta$ prc-RS218

We further investigated whether inactivation of the $\sigma^{\mathrm{E}}$ or RcsCDB systems could restore the expression of $f l h D C$ and $\mathrm{FliC}$ in the prc mutant. yiiS and $y j b E$ are the genes positively regulated by the $\sigma^{\mathrm{E}}$ and $\mathrm{RcsCDB}$ systems, respectively. Thus, the expression levels of these genes can reflect the activation levels of the corresponding signaling systems [51, 52]. In comparison with $\Delta p r c-\mathrm{RS} 218$, the double mutants $\Delta$ degs $\Delta p r c$-RS218 and $\Delta r c s B \Delta p r c$-RS218 showed lower expression of yiiS and yjbE (Fig. 9a and b). This result indicates that the prc deletion-induced $\sigma^{\mathrm{E}}$ and RcsCDB activation was blocked in $\Delta$ degs $\Delta p r c$-RS218 and $\Delta r c s B \Delta p r c-\mathrm{RS} 218$, respectively. In addition, in comparison with $\Delta p r c$-RS218, both $\Delta$ degs $\Delta p r c$-RS218 and $\Delta r c s B \Delta p r c$ RS218 showed increased levels of flhDC (Fig. 9a and b) and FliC expression (Fig. 9c and d). These results suggest that the activated $\sigma^{\mathrm{E}}$ or RcsCDB systems contribute to the suppressed flagellar expression, resulting in the defective motility in the prc mutant.

\section{The activated $\sigma^{\mathrm{E}}$ system suppresses motility and flhDC transcription in wild-type ExPEC}

It has been previously shown that activation of the RcsCDB system can downregulate the expression of $f h D C$ and thus suppress motility in E. coli without prc mutation [21, 24]. This finding suggests that the activation of the RcsCDB signaling pathway alone in the prc mutant strain is sufficient to cause the suppression, requiring no other signals induced by the prc mutation. To the best of our knowledge, this report is the first study demonstrating that the $\sigma^{\mathrm{E}}$ system is involved in motility suppression in the prc mutant of $E$. coli (Fig. 8a, b, and d). However, it remained unclear whether activation of the $\sigma^{E}$ system could suppress bacterial motility in an E. coli strain with an intact Prc. We assessed the motility and $f l h D C$ expression of a $\operatorname{prc}^{+} \mathrm{RS} 218$ strain $(\Delta r s e A$ RS218) in which the $\sigma^{\mathrm{E}}$ system was activated by deleting the anti- $\sigma^{\mathrm{E}}$ factor RseA [58]. As shown in Fig. 10a and b, activation of the $\sigma^{\mathrm{E}}$ system in the prc $^{+}$background

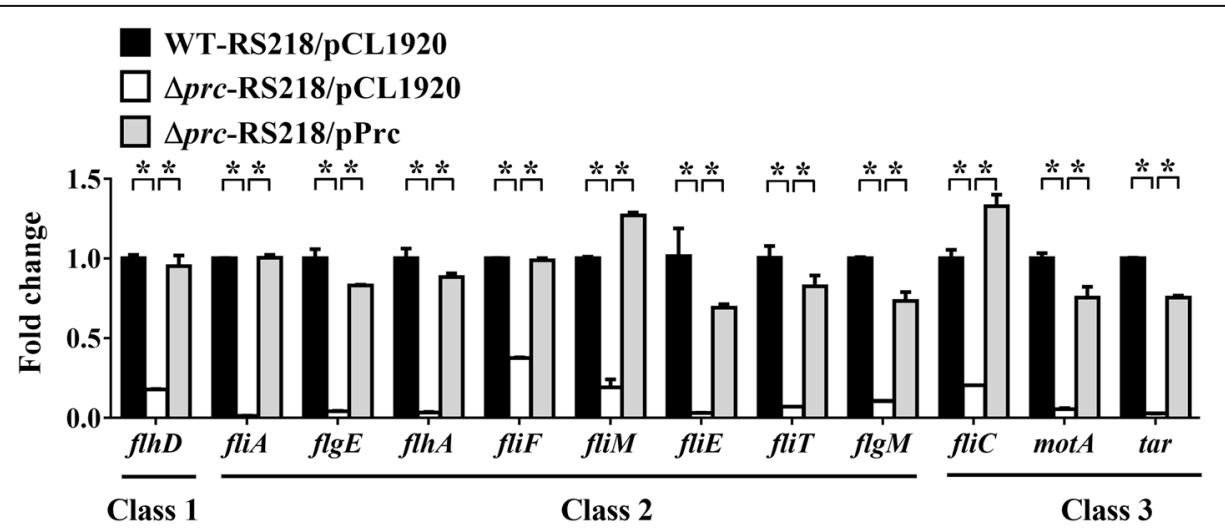

Fig. 4 The expression of the flagellar regulon in the wild-type, prc mutant, and complemented strains of ExPEC. The transcript levels of the class 1 gene flhD, class 2 genes (fliA, flgE, flhA, fliF, fliM, fliE, fliT and flgM) and class 3 genes (flic, motA, and tar) were determined by qPCR. The transcript levels of the genes in each strain, which were normalized to those of the housekeeping gene $\mathrm{fts} Z$, are presented as the relative levels compared to those in WT-RS218/pCL1920. The results were derived from experiments performed in triplicate and are shown as the means \pm standard deviations. pCL1920, the empty plasmid vector; pPrc, the plasmid pCL1920 harboring prc (Table 1). The asterisks indicate significant differences ( $P$ values $<0.05$ ) of the comparisons between WT-RS218/pCL1920 and $\Delta$ prc-RS218/pCL1920 as well as between $\Delta$ prc-RS218/pCL1920 and $\Delta$ prc-RS218/pPrc 


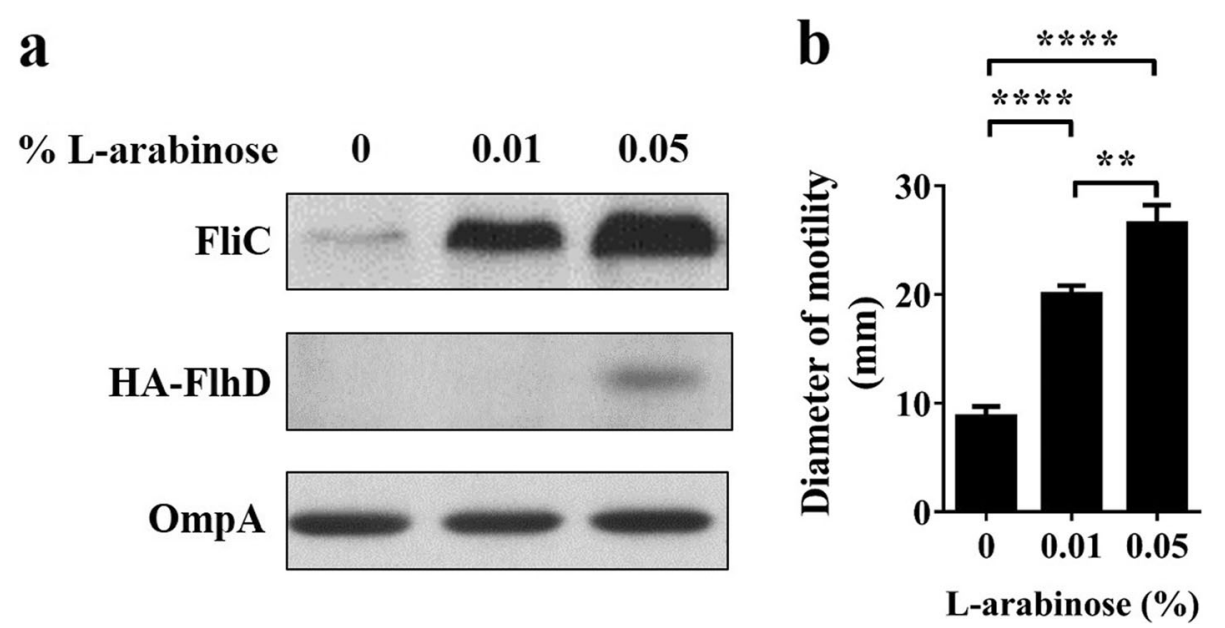

Fig. 5 Effects of increased FlhDC expression on the FliC level and motility in the prc mutant. FliC and HA-tagged FlhD levels (a) and the motility (b) of $\triangle p r c-R S 218 / p B A D-F l h D C$ with different levels of L-arabinose treatment. Each motility quantitative result was derived from experiments performed in triplicate and is presented as the means \pm standard deviations. The plasmid pBAD-FlhDC harbored HA-tagged flhD and His-tagged flhC genes that were under the control of an arabinose-inducible promoter (Table 1). The levels of FliC, FlhD, and OmpA were determined by western blot analyses with a rabbit anti-FliC antiserum and an anti-HA antibody and mouse anti-OmpA antiserum, respectively. The levels of OmpA served as loading controls

significantly decreased bacterial motility and suppressed flhDC expression. These findings suggested that without other prc deletion-induced signals, activation of the $\sigma^{\mathrm{E}}$ system was sufficient to suppress $f l h D C$ expression and thus bacterial motility in the prc mutant.

The $\sigma^{\mathrm{E}}$ and RcsCDB systems can work independently to downregulate bacterial motility

We investigated whether the activation of the $\sigma^{\mathrm{E}}$ and RcsCDB systems in ExPEC are sequential (upstream and downstream) events. It has been shown that RcsB overexpression triggers the activation of the RcsCDB system [59]. RS218 was transformed with the RcsB-overexpressing plasmid pRcsB (WT-RS218/pRcsB) to create a RcsCDBactivated strain. WT-RS218/pRcsB showed significantly higher $y j b E$ expression than RS218 harboring an empty plasmid vector (WT-RS218/pBAD), while the two strains showed similar levels of yiiS expression (Fig. 11a). This result suggested that activating the RcsCDB system does not trigger the activation of the $\sigma^{\mathrm{E}}$ system. On the other hand, the $\sigma^{\mathrm{E}}$-activated strain $\Delta r s e A$-RS218 showed significantly higher yiiS expression than WT-RS218, while these two strains showed similar levels of $y j b E$ expression (Fig. 11b). This finding suggested that activation of the $\sigma^{\mathrm{E}}$ system does not trigger the activation of the RcsCDB system. Collectively, these results suggested that the activation of these systems is independent of each other in ExPEC.

We further determined whether the two systems are also independently activated by Prc deficiency. $\Delta r c s B \Delta p r c-$ $\mathrm{RS} 218$, in which the activation of the RcsCDB system was blocked, and $\Delta p r c$-RS218 showed a similar level of yiiS expression (Fig. 11c), suggesting that the RcsCDB signal does not contribute to the activation of the $\sigma^{\mathrm{E}}$ system in the prc mutant. The $\sigma^{\mathrm{E}}$-inactivated strain $\Delta$ degS $\Delta p r c$-RS218 showed a slightly higher level of $y j b E$ expression than $\Delta p r c$ RS218 (Fig. 11d), suggesting that $\sigma^{\mathrm{E}}$ does not contribute to RcsCDB system activation in the prc mutant. Taken together, these results indicate that Prc deficiency independently induces the activation of the $\sigma^{\mathrm{E}}$ and $\mathrm{RcsCDB}$ systems.

\section{The protease activity of Prc is required for bacterial motility and FliC expression}

Since Prc is a protease, we investigated whether the deficiency of the Prc protease function contributes to the decreased motility and FliC expression in the prc mutant. Prc variants with a S430A or K455A substitution are known to lose catalytic activity but still maintain the original protein structure and substrate-binding ability [60]. Trans-complementation of $\Delta p r c-$ RS218 with the catalytic ability-defective variants failed to restore the motility and FliC level (Fig. 12a and b). These results suggested that deficiency in the Prc protease activity is responsible for the reduced flagellum expression and thus the defective motility.

The accumulation of Spr is responsible for the defective motility and decreased FliC expression in $\Delta$ prc-RS218 Given that a protease exerts biological function through mainly proteolytic regulation of its substrates, we reasoned that the dysregulation of Prc substrates due to the deficiency in the protease activity may be responsible for the reduced motility in the prc mutant. The 


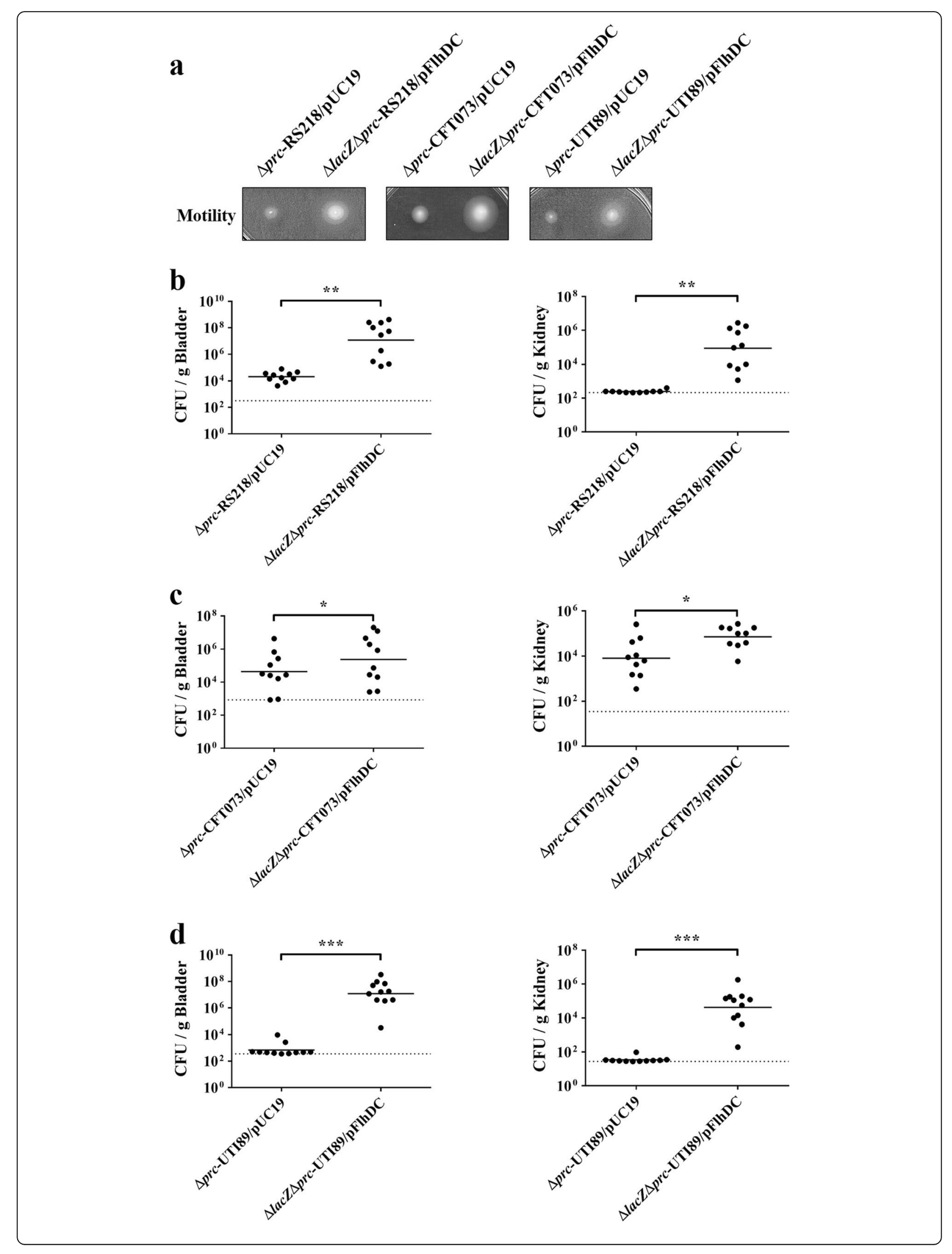


Fig. 6 Effect of motility increase on the ability of the ExPEC prc mutants to cause UTIs. (a) Construction of prc mutants constitutively overexpressing flhDC. The $\triangle$ lacZ $\Delta$ prc strains of the ExPEC strains RS218, CFT073, and UTI89 were transformed with the plasmid pFlhDC (Table 1). The flhDC operon encoded in this plasmid was fused with a lac promoter. Without any induction, the flhDC overexpression driven by leaky promoter activity was strong enough to improve the bacterial motility of the prc mutants. (b, $\mathbf{c}$, and $\mathbf{d}$ ) Transurethral cochallenge of mice with the prc mutants of the indicated ExPEC strains with and without flhDC overexpression. At $48 \mathrm{hpi}$, the bacterial counts in the bladders and kidneys were enumerated and differentiated by spreading the homogenized infected tissue onto LB agar plates with IPTG and X-gal. Horizontal bars indicate the median level of the bacterial counts. The dotted line represents the limit of detection

Prc substrate Spr has been shown to accumulate in a prc mutant of the commensal E. coli MG1655 [38]. Similarly, our proteome data showed that $\Delta p r c$-RS218 expressed a significantly higher level of Spr than WTRS218 (Table 2). A western blot analysis of Spr in the RS218 strains with or without prc further confirmed this finding (Fig. 13a), suggesting that Spr accumulation is common among the E. coli strains in which Prc is inactivated. We overexpressed Spr to raise the intracellular level of Spr in RS218. Spr overexpression

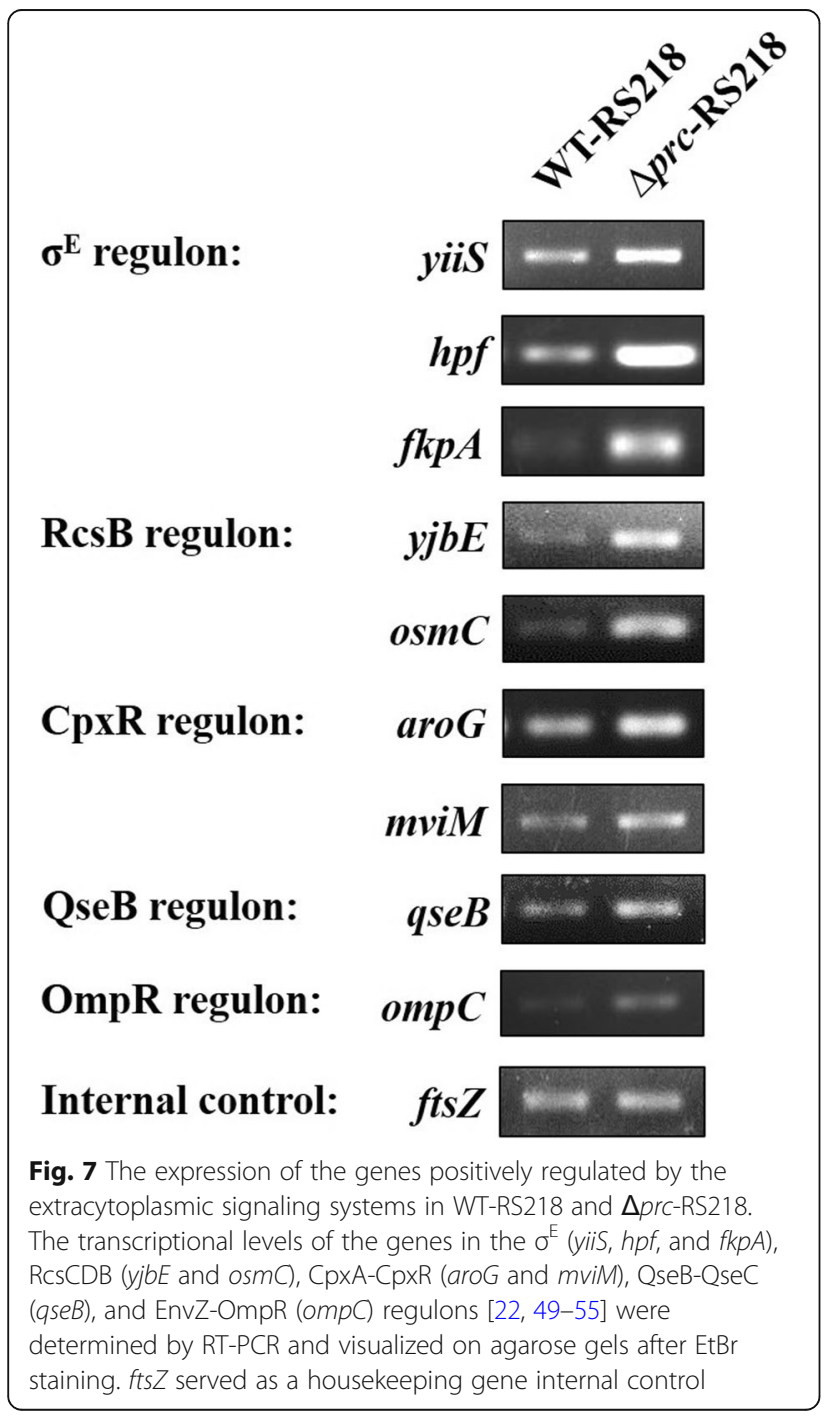

significantly decreased the FliC level (Fig. 13b) and bacterial motility (Fig. 13c), suggesting that Spr accumulation in the prc mutant contributes to reduced motility.

To determine whether Spr accumulation in ExPEC triggers activation of the RcsCDB and $\sigma^{\mathrm{E}}$ systems, the transcription levels of the RcsCDB-regulated $y j b E$ and $\sigma^{\mathrm{E}}$ regulated yiiS genes in an Spr-overexpressing strain (WTRS218/pSpr) and a strain with normal Spr expression (WTRS218/pBAD) were determined (Fig. 13d). Overexpression of Spr upregulated $y j b E$, but $y i i S$ was not affected. This suggested that $\mathrm{Spr}$ accumulation triggers activation of the RcsCDB system but not the $\sigma^{\mathrm{E}}$ system. Consistent with this finding, blocking the activation of RcsCDB signaling by deleting $r c s B$ significantly increased the motility of the Sproverexpressing strain, while blocking $\sigma^{\mathrm{E}}$ signaling by deleting $\operatorname{deg} S$ did not affect bacterial motility (Fig. 13c). Additionally, deletion of $r c s B$ increased the FliC level in the Spr-overexpressing strain (Fig. 13b). These results suggest that $\mathrm{Spr}$ accumulation in the prc mutant triggers activation of the RcsCDB system and thus suppresses flagellin expression and bacterial motility.

In addition, we further investigated whether blocking Spr accumulation in the prc mutant affects the activation of the RcsCDB system and motility. We blocked Spr accumulation in the prc mutant by deleting the $s p r$ gene ( $\Delta s p r \Delta p r c-R S 218) . \quad$ Similar to wild-type RS218, $\Delta s p r \Delta p r c-R S 218$ showed significantly lower yibE expression than $\Delta p r c-$ RS218 (Fig. 13e), further supporting that Spr accumulation results in the activation of the RcsCDB system in the prc mutant. However, the FliC level (Fig. 13f) and motility (Fig. 13g) of $\Delta s p r \Delta p r c-R S 218$ were not significantly higher than those of $\Delta p r c$-RS218. These results suggest that deleting spr in the prc mutant may cause other pleotropic effects that are able to downregulate FliC expression and motility, which can offset the effects of the downregulated RcsCDB activation.

\section{Discussion}

This study reveals for the first time that the periplasmic protease Prc in ExPEC contributes to the pathogenesis of UTIs through maintaining intact bacterial motility, which is required for ExPEC to colonize the bladder and kidney [12-15]. In addition, the mechanism of how Prc deficiency interferes with the motility in ExPEC has been elucidated (see the model in Fig. 14). Lack of $\operatorname{Prc}$ in the bacteria triggers $\sigma^{\mathrm{E}}$ and 


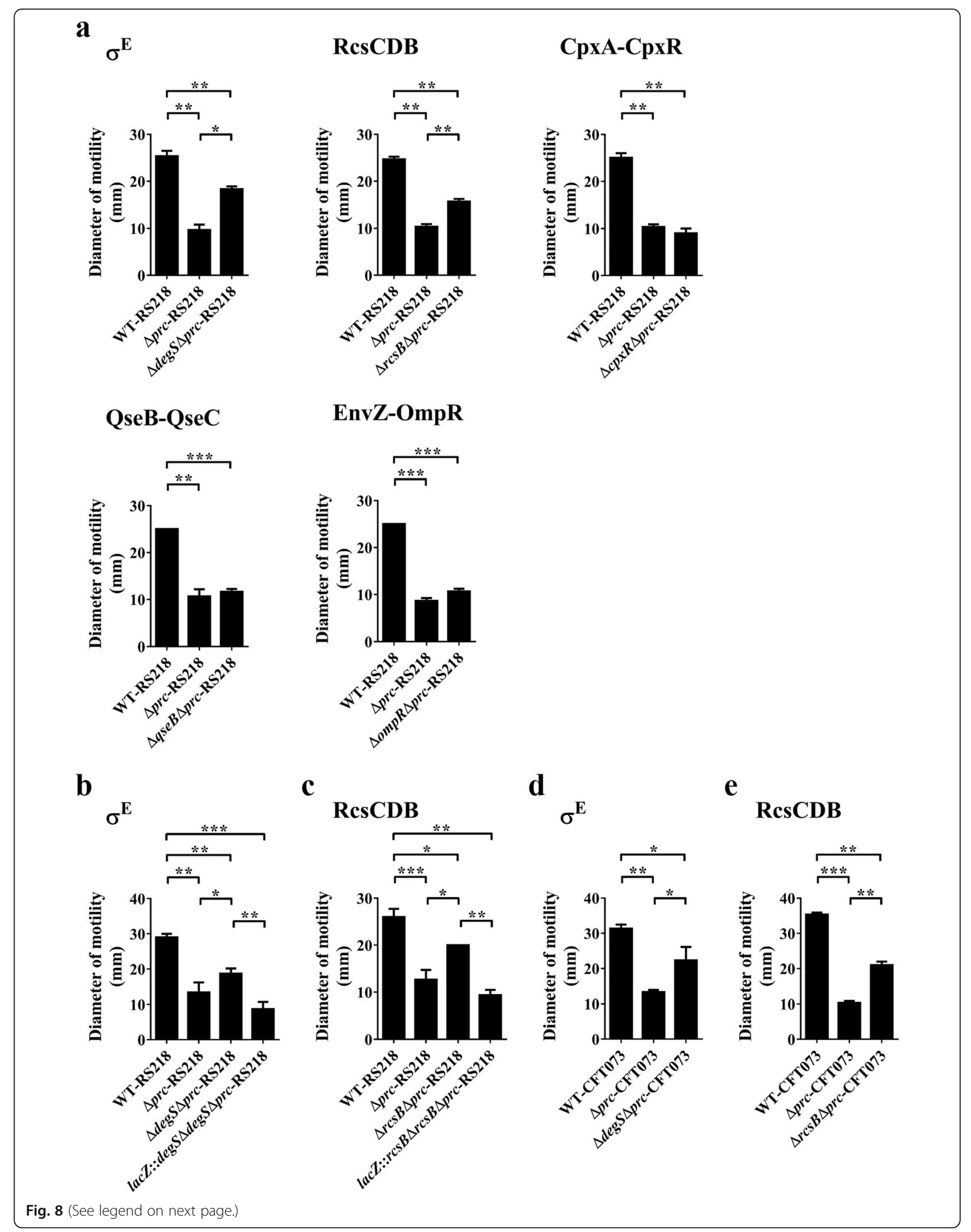


(See figure on previous page.)

Fig. 8 The motilities of the prc mutant strains with inactivated extracytoplasmic signaling pathways. (a) The motilities of the prc mutant strains of RS218 with a blocked $\sigma^{E}$, RcsCDB, CpxA-CpxR, QseB-QseC, or EnvZ-OmpR system. degS, rcsB, cpxR, aseB, and ompR deletions were introduced into $\Delta$ prc-RS218 to block the $\sigma^{E}$, RcsCDB, CpxA-CpxR, QseB-QseC, and EnvZ-OmpR signaling pathways, respectively. (b) The motility of the $\Delta$ degs $\Delta p r c-$ RS218 strain complemented with degS in the chromosomal lacZ locus. (c) The motility of the $\Delta$ rcsB $\Delta$ prc-RS218 strain complemented with rcsB in the chromosomal lacZ locus. (d and e) The motilities of the prc mutant strains of CFT073 with an inactivated $\sigma^{\mathrm{E}}$ (d) or RcsCDB (e) system

RcsCDB signaling, which in turn negatively regulate the expression of the master operon $f l h D C$ of the flagellar regulon, leading to decreased flagellum expression and hindered bacterial motility. To our knowledge, this report is also the first study demonstrating that $\sigma^{\mathrm{E}}$ signaling negatively regulates the expression of flagella, while activated RcsCDB signaling has previously been shown to be able to suppress this bacterial structure [21]. The accumulation of Spr, a Prc substrate, is responsible for the activated RcsCDB signaling in the ExPEC prc mutant. These results demonstrate that Prc-mediated proteolytic regulation of the intracellular substrate is critical for sufficient ExPEC motility to cause UTIs.

The RcsCDB system monitors damage of the OM and peptidoglycan layer [24, 61-64]. It is highly likely that the uncontrolled Spr level in the prc mutant activates the RcsBCD system through altering the peptidoglycan structure and interfering with $\mathrm{OM}$ integrity. This notion is consistent with the known physical function of Spr and the phenotype resulting from the overexpression of $\mathrm{Spr}$ in E. coli $[65,66]$. Spr is an OM protein [67] and a peptidoglycan hydrolase involved in maintaining the stability of the bacterial peptidoglycan structure [65]. It has been shown that overexpression of $\mathrm{Spr}$ in E. coli interferes with peptidoglycan biogenesis, leading to decreased peptidoglycan crosslinkage with the $\mathrm{OM}$, which consequently destabilizes the bacterial envelope [66]. In addition, the high level of Spr located on the OM may be a contributing factor to the compromised $\mathrm{OM}$ integrity in the prc mutant of ExPEC.
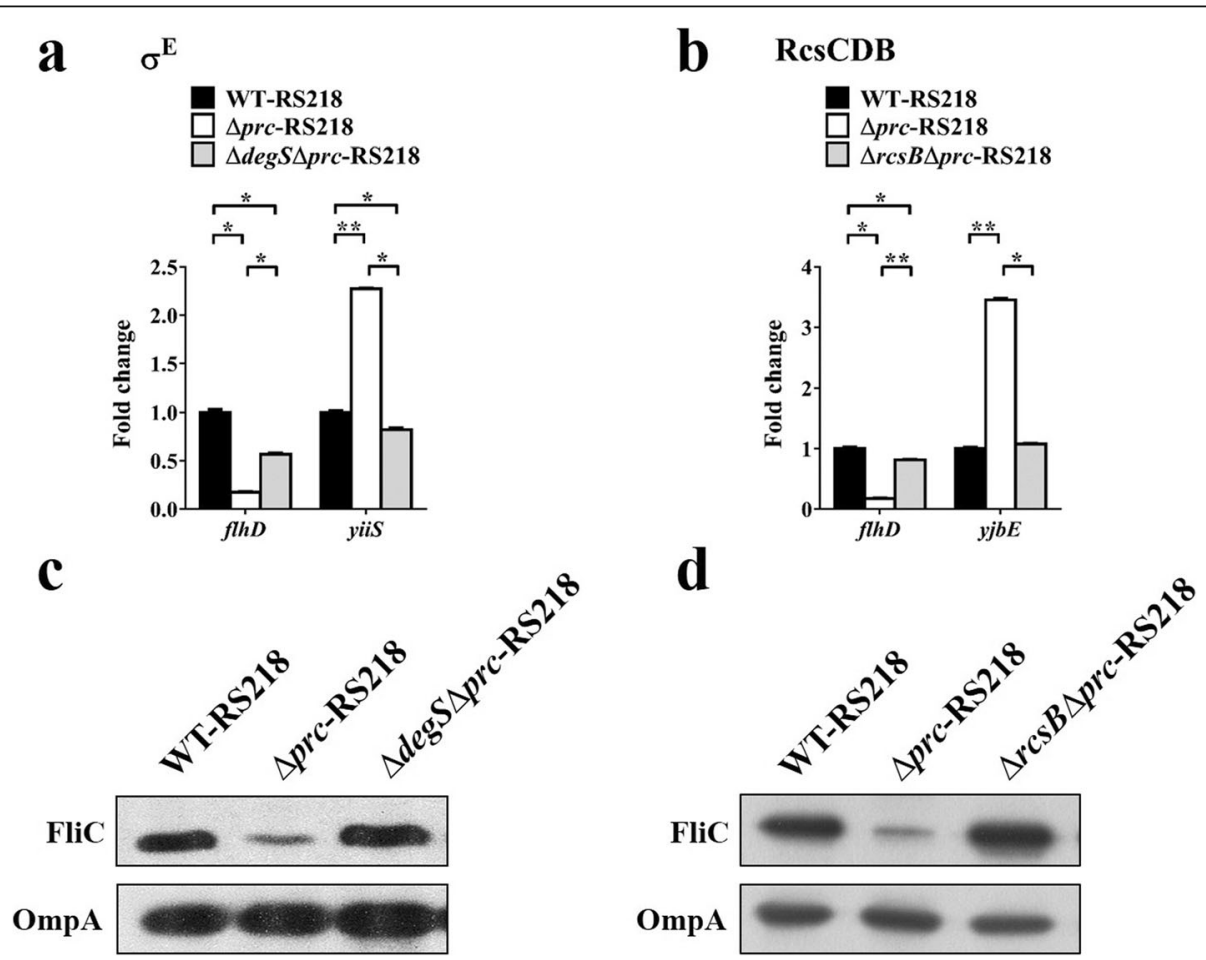

Fig. 9 The effects of $\sigma^{E}$ and RCSCDB inactivation on the transcript levels of the flhDC operon and the FliC levels in the prc mutant. (a and $\mathbf{b}$ ) The mRNA levels of flhD, yiiS, and yjbE in the indicated strains determined by qPCR. The levels of yiis and yjbE reflect the activation levels of the $\sigma^{E}$ and RcsCDB systems, respectively. The mRNA level of each gene in a strain was normalized to the fts $Z$ level and presented as a relative level compared to that in WT-RS218. The results were derived from experiments performed in triplicate and are shown as the means \pm standard deviations. (c and $\mathbf{d}$ ) The levels of FliC in the indicated strains were determined by western blot analysis with a rabbit anti-FliC antiserum. OmpA levels served as loading controls 

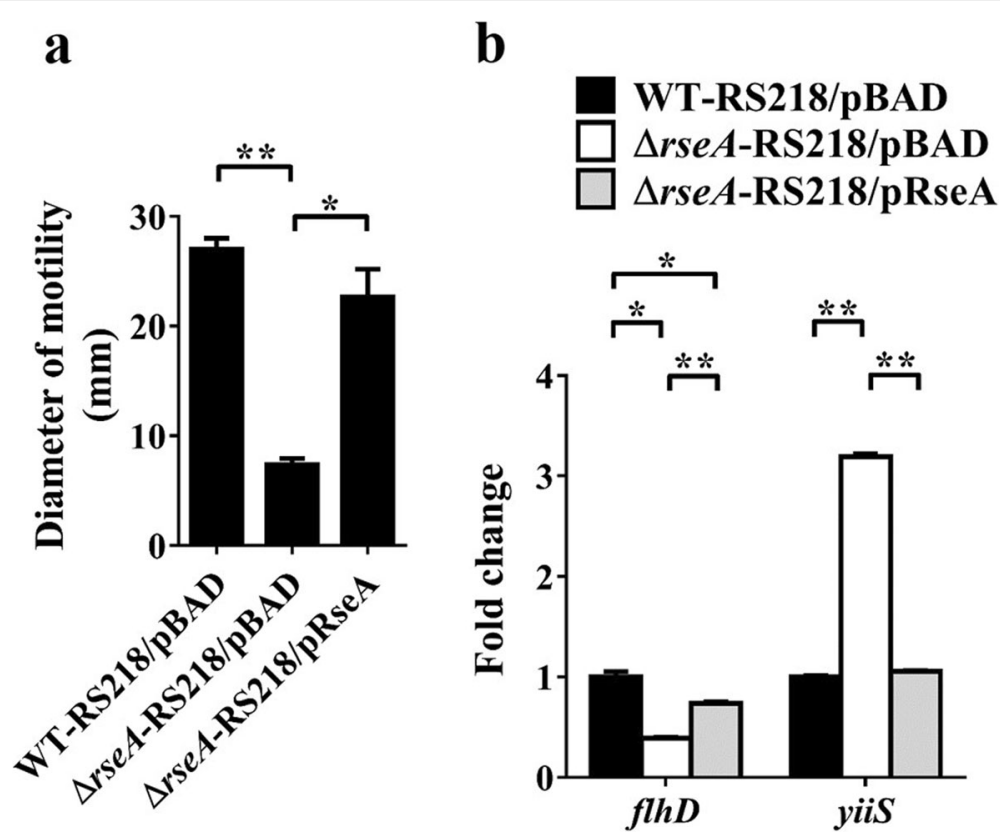

Fig. 10 The effect of $\sigma^{\mathrm{E}}$ activation on the motility and transcript level of flhD in the prc ${ }^{+}$ExPEC strain. (a) Motility diameters of the indicated strains. (b) Relative mRNA levels of flhD and yiiS determined by qPCR. The yiiS gene served as the reporter of $\sigma^{E}$ activation. The mRNA level of each gene in a strain was normalized to the ftsZ level and presented as a relative level compared to that in WT-RS218 harboring pBAD (WTRS218/pBAD). Arabinose (0.2\%) was used to induce the expression of RseA that was encoded in the pRseA plasmid (Table 1) and driven by the arabinose-inducible promoter on the plasmid. The results were derived from experiments performed in triplicate and are shown as the means \pm standard deviations. pRseA, pBAD harboring rseA

Dual molecular signals are required to activate the $\sigma^{\mathrm{E}}$ signaling system: the periplasmic accumulation of denatured OMPs and LPS [36, 68]. Thus, the activation of this signaling system in the prc mutant suggests that Prc deficiency causes the accumulation of denatured OMPs and LPS in the periplasmic space. In agreement with this hypothesis, we found that overexpression of DegQ, which is a periplasmic chaperone able to renature misfolded proteins in the periplasm [69], could downregulate the $\sigma^{\mathrm{E}}$ activation and upregulate the FliC expression and motility of the E. coli prc mutant (Additional file 4: Figure S1). In addition, construction of the $\mathrm{OM}$ requires the transport of OMPs and LPS through the periplasmic space into the OM. In the prc mutant, the periplasmic accumulation of these components suggests that Prc deficiency may hinder their transport from the periplasm to the OM. The global proteomic analysis showed that the prc mutant ( $\Delta p r c$-RS218) exhibited a significantly higher level of BamA expression than the wild-type strain (Table 2). BamA is the essential component of the $\beta$-barrel protein assembly machinery (BAM complex), which is responsible for the assembly of OMPs and the LPS transporter proteins in the OM $[70,71]$. The increased BamA expression may be a compensatory response for the mutant since the complex is required for the transport of the OMPs and LPS transporters from the periplasm to the OM.

In the prc mutant, both the RcsCDB and $\sigma^{\mathrm{E}}$ systems suppressed bacterial motility through downregulating the expression of $f l h D C$. It has been known that RcsB can directly bind to the promoter of $f l h D C$ to suppress its transcription when the RcsCDB system is activated [21]. However, how $\sigma^{\mathrm{E}}$ signaling downregulates this operon remains to be elucidated. The RNA polymerase holoenzyme (holo-RNAP) is composed of an $\sigma$ subunit and a core RNA polymerase (co-RNAP), which are responsible for promoter selectivity and RNA synthesis, respectively. In bacterial cells, different types of $\sigma$ factors compete for a limited pool of common co-RNAP to transcribe a set of genes driven by their cognate promoters [72]. Transcription of the $f l h D C$ operon is dependent on the housekeeping sigma factor $\sigma^{70}$ [73]. It is likely that in the prc mutant, the activated $\sigma^{\mathrm{E}}$ system allows the $\sigma^{\mathrm{E}}$ factor to compete with $\sigma^{70}$ for co-RNAP, thus resulting in the downregulation of the $f l h D C$ operon. Alternatively, $\sigma^{\mathrm{E}}$ may trigger the expression of unknown bacterial factors capable of downregulating $f l h D C$ transcription.

The present study demonstrates that constitutive activation of $\sigma^{\mathrm{E}}$ and RcsCDB signaling attenuates ExPEC in UTIs through suppressing flagellum-mediated motility. However, it has been shown that blocking $\sigma^{\mathrm{E}}$ signaling 
a

\section{WT-RS218/pBAD WT-RS218/pResB}

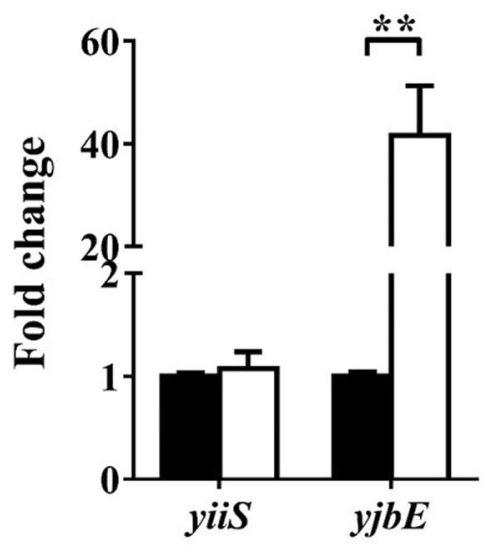

c

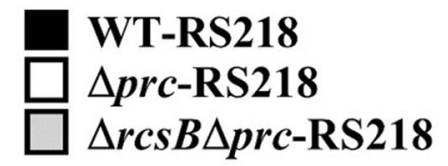

b

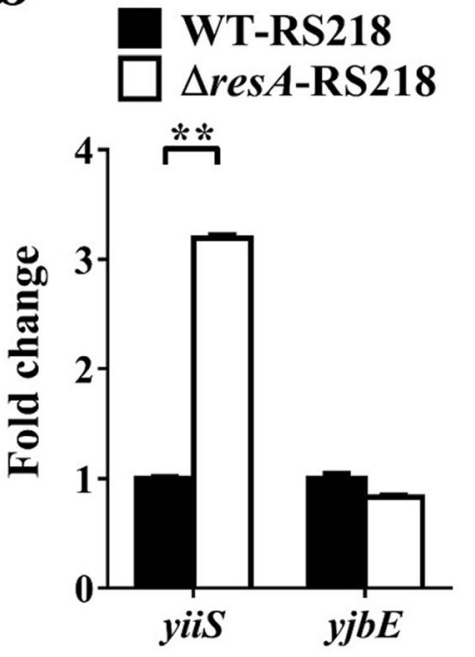

d
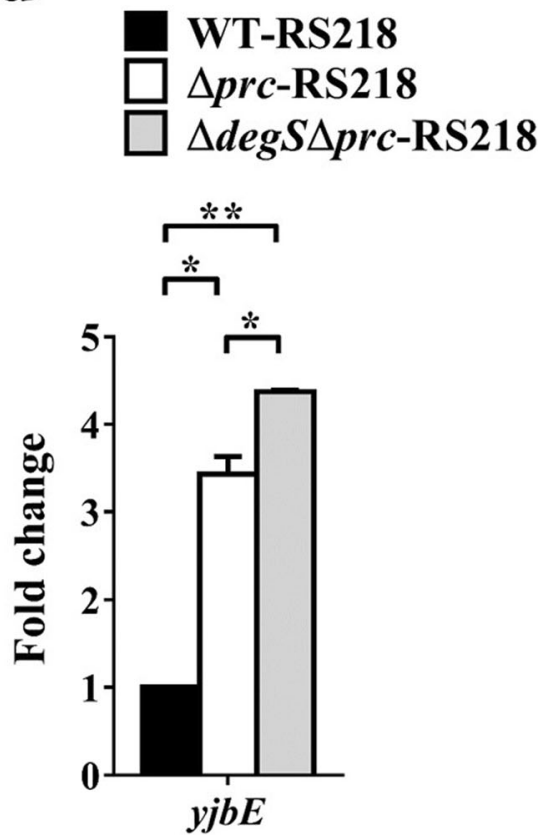

Fig. 11 The activated $\sigma^{E}$ and RcsCDB systems can work independently to suppress bacterial motility. (a) The mRNA levels of the $\sigma^{E}$-regulated gene yiiS and the RCSCDB-regulated gene yjbE in the RS218 strain with an unactivated or activated RcsCDB system. Arabinose (0.2\%) was used to induce the overexpression of RcsB encoded in PRcsB (Table 1). (b) The mRNA levels of yiil and yjbE in the bacteria with an unactivated or activated $\sigma^{E}$ system. (c) The mRNA levels of yiiS in WT-RS218, $\Delta$ prc-RS218, and $\Delta r c s B \Delta$ rc-RS218. (d) The mRNA levels of yjbE in WT-RS218, $\Delta p r c-$ RS218, and $\Delta$ degS $\Delta$ prc-RS218. The mRNA level of each gene, which was determined by qPCR, in a strain was normalized to the ftsZ level and presented as a relative level compared to that in WT-RS218. The results were derived from experiments performed in triplicate and are shown as the means \pm standard deviations. pRcsB, pBAD harboring the rcsB gene driven by the arabinose-inducible promoter on the plasmid

will also reduce the bacterium's ability to cause UTIs [74]. These findings suggest that a tunable $\sigma^{\mathrm{E}}$ signaling system rather than a constantly activated or silenced one is required for the whole virulence of the pathogen during infections. To cause UTIs, ExPEC need to accomplish multiple pathogenic steps, such as adhering to the epithelium lining of the UT, disseminating within the UT, and evading the host immune responses, etc. [75]. The existence of a certain bacterial factor may benefit one pathogenic step but hinder another. Strict 

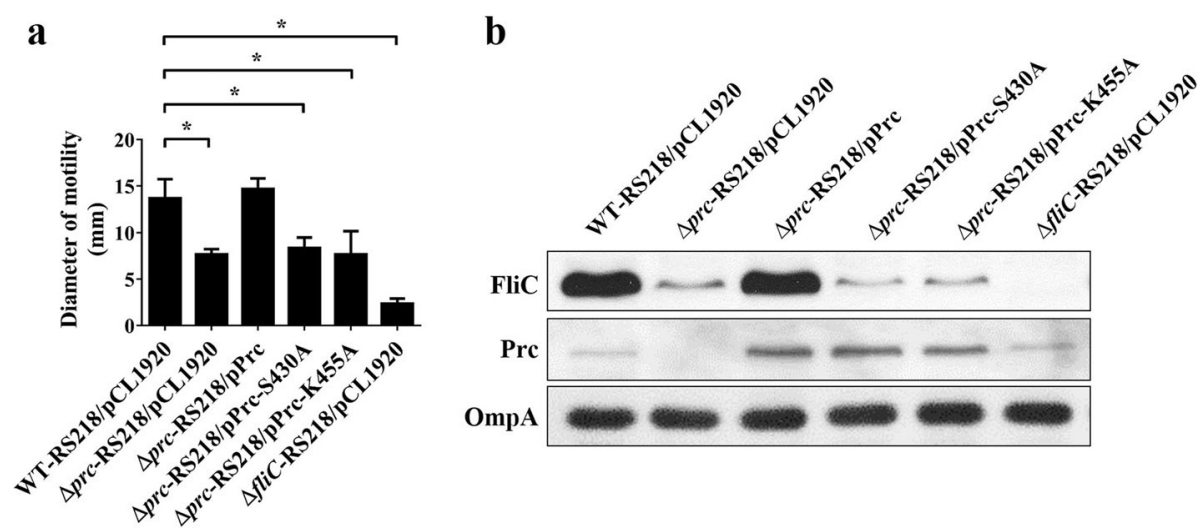

Fig. 12 A lack of Prc protease activity was responsible for the defective motility and decreased FliC expression in the prc mutant. (a) Motility diameters of the strains expressing wild-type Prc, Prc S430A, and Prc K455A. The results were derived from three independent experiments and are shown as the means \pm standard deviations. (b) The protein levels of FliC, Prc, and OmpA in the bacteria expressing wild-type Prc, Prc S430A, and $\operatorname{Prc}$ K455A. The protein levels were determined by western blot analysis with a rabbit anti-FliC antiserum, rabbit anti-Prc antiserum and mouse anti-OmpA antiserum, respectively. The OmpA level served as a loading control. $\Delta$ flic/pCL1920 served as a nonmobile and non-FliC expression control. pCL1920, the plasmid pCL1920, which served as an empty vector control. pPrc, the plasmid pCL1920 harboring prc; pPrcS430A, the plasmid pCL1920 harboring a mutated prc expressing Prc S430A; PPrc-K455A, the plasmid pCL1920 harboring a mutated pre expressing Prc K455A

regulation of the expression of such factors may also be required for the whole virulence of the pathogen. While flagella are necessary for $E$. coli to disseminate within the UT during infections, flagellin, the major component of flagella, is a potent immunogen able to activate the immune response via TLR5, which may cause the clearance of the invading pathogens. Therefore, $\sigma^{\mathrm{E}}$ signaling may need to remain tunable so that it can work in coordination with flagellar expression to achieve a successful UTI. In addition, inactivation of the RcsCDB system has been shown to decrease the ability of ExPEC resistance to serum-mediated killing [76]. This finding suggest that a tunable RcsCDB system may also be essential for the full virulence of ExPEC. Thus, hindering the proper function of the $\sigma^{\mathrm{E}}$ and RcsCDB signaling systems would be a potential strategy to fight against the bacterial infections.

The decreased motility may not be fully responsible for the defective ability of the ExPEC prc mutant to cause UTIs. Our previous study demonstrated that prc deletion decreases the resistance of ExPEC to complement-mediated killing in the bloodstream [4]. It has been suggested that pathogenic E. coli could be opsonized by the complement system in the UT [77]. Since bacterial opsonization could facilitate phagocytosis by phagocytes, leading to the elimination of invading bacteria in the tissue, defective resistance to the complement system may also attenuate the prc mutant in UTIs. In agreement with this hypothesis, we found that increasing the motility of $\Delta p r c$-RS218 by overexpressing $f l h D C$ to the level of $f l h D C$ expression in WT-RS218 could not restore the mutant's UT colonization to the level of the wild-type strain (data not shown). On the other hand, the decreased motility was not responsible for the prc mutant's defect in the resistance to killing mediated by the complement system because increasing the motility of $\Delta p r c$-RS218 did not restore bacterial resistance to complement-mediated serum killing (data not shown).

In addition to FliC, several other outer membrane proteins whose expression levels were significantly affected by the prc deletion were found in the LC/MS/ MS analysis (Table 2). The altered expression levels of the proteins may also contribute the attenuated virulence of the ExPEC prc mutant. For example, SlyB was shown to be downregulated in the prc mutant of RS218 (Table 2). It is known that deletion of SlyB in Burkholderia multivorans attenuates bacterial iron uptake ability and compromises the OM integrity [78]. Given that the iron uptake ability and an intact OM integrity are important for pathogenic bacteria to invade hosts [79-81], it is worth further investigating whether the downregulated SlyB expression in the ExPEC prc mutant decreased the bacterial ability to cause UTIs. In addition, Tsx was shown to be upregulated in the prc mutant of RS218. The outer membrane porin Tsx is essential for E. coli to uptake of a gyrase inhibitor antibiotic, albicidin [82-84]. It has been known that deletion of prc increases the susceptibilities of $E$. coli to multiple antibiotics [85]. The increased Tsx level may be one of the contributing factors. 

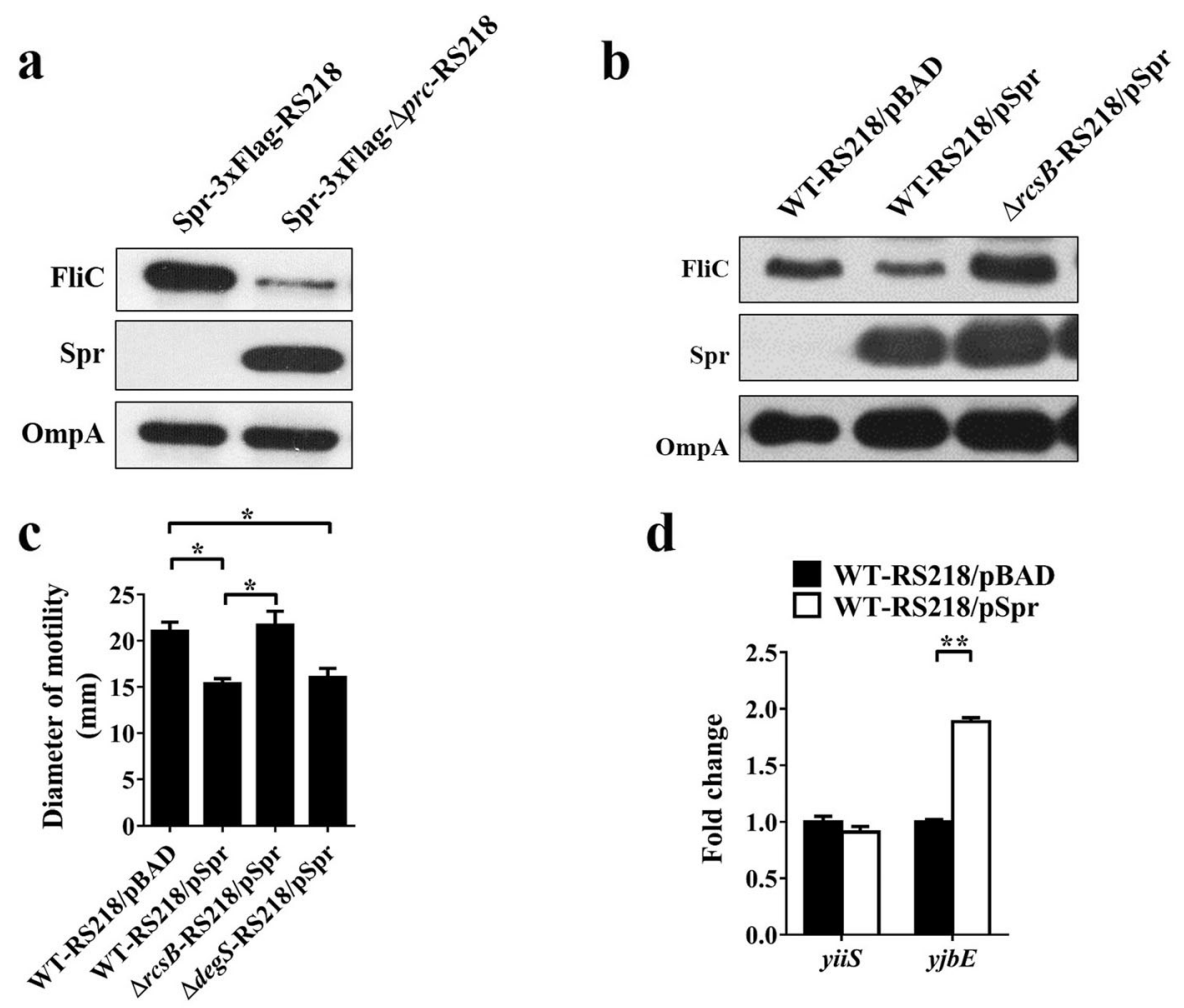

d
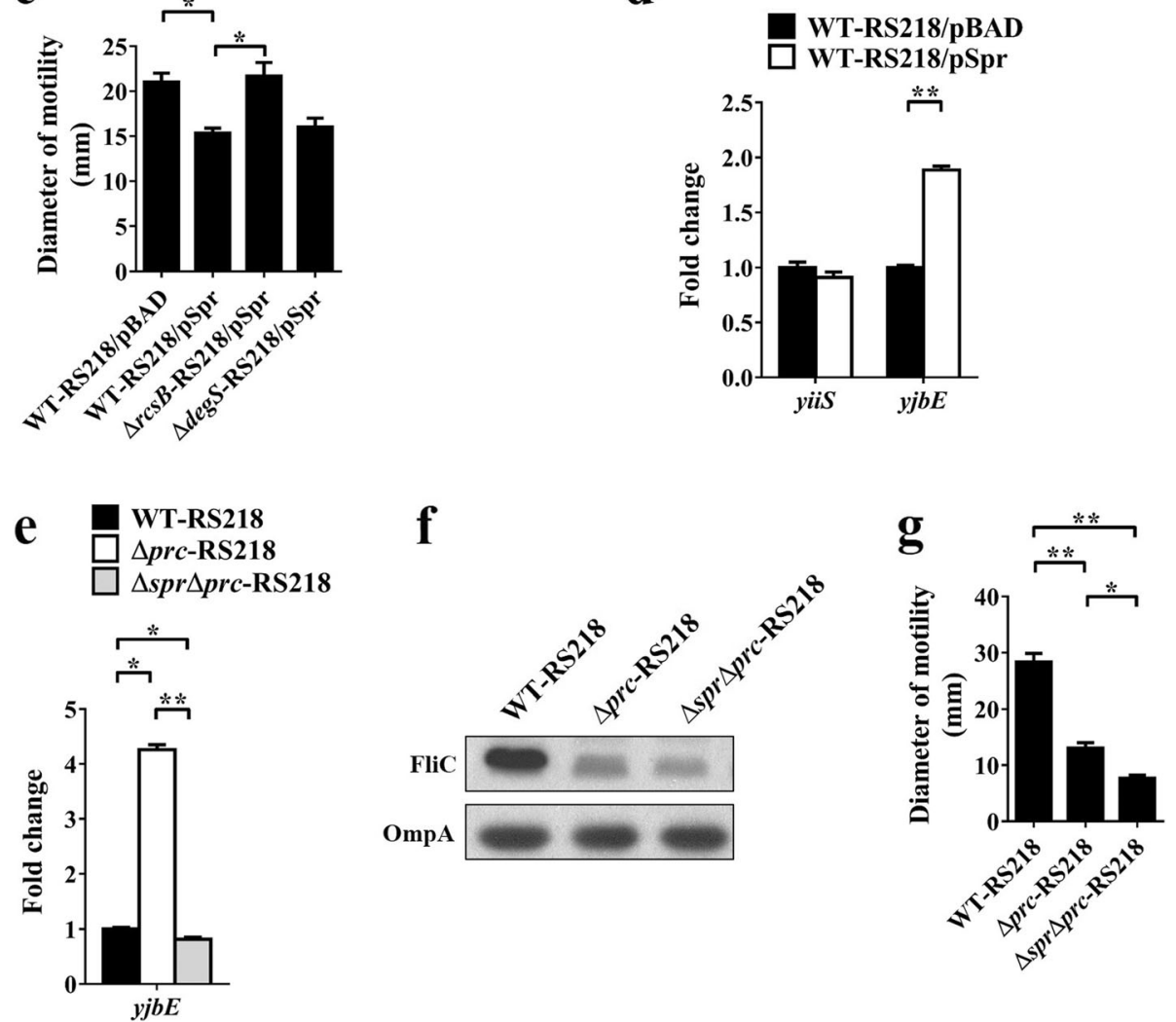

Fig. 13 Effects of Spr accumulation on bacterial motility, FliC expression and the activation of the extracytoplasmic signaling systems. (a) The levels of Spr and FliC in the RS218 strains with or without prc. To detect Spr, these strains were modified to express C-terminally 3xFlag-tagged Spr. Western blot analyses with an anti-Flag antibody and a rabbit anti-FliC antiserum were performed to detect the protein levels. The OmpA levels served as loading controls, which were probed with a mouse anti-OmpA antiserum. Spr-3xFlag-RS218, the RS218 strain with the native chromosomal spr fused with a sequence encoding a 3xFLAG tag at the $3^{\prime}$ end; Spr-3xFlag- $\Delta$ prc-RS218, the $\Delta$ prc-RS218 strain with the chromosomal spr fused with a sequence encoding a 3xFLAG tag at the $3^{\prime}$ end. (b) The FliC and Spr levels in the RS218 strains with or without the overexpression of the recombinant Spr, which was C-terminally fused with a Flag tag. The Spr protein was detected with an anti-Flag antibody. Arabinose (0.2\%) was used to trigger the expression of the recombinant Spr that was encoded in pBAD and driven by the arabinoseinducible promoter in the plasmid. pSpr, pBAD harboring spr fused with a sequence encoding a Flag tag at the 3' end. (c) Motility diameters of the strains overexpressing Spr. (d) The relative mRNA levels of yiiS and yjbE compared to those in WT-RS218/pBAD. (e) The relative yibE levels compared to those in WT-RS218. (f) The FliC level in WT-RS218, $\Delta$ prc-RS218, and $\Delta$ spr $\Delta$ prc-RS218. (g) Motility diameters of WT-RS218, $\Delta$ prc-RS218, and $\Delta$ spr $\Delta$ prc-RS218 


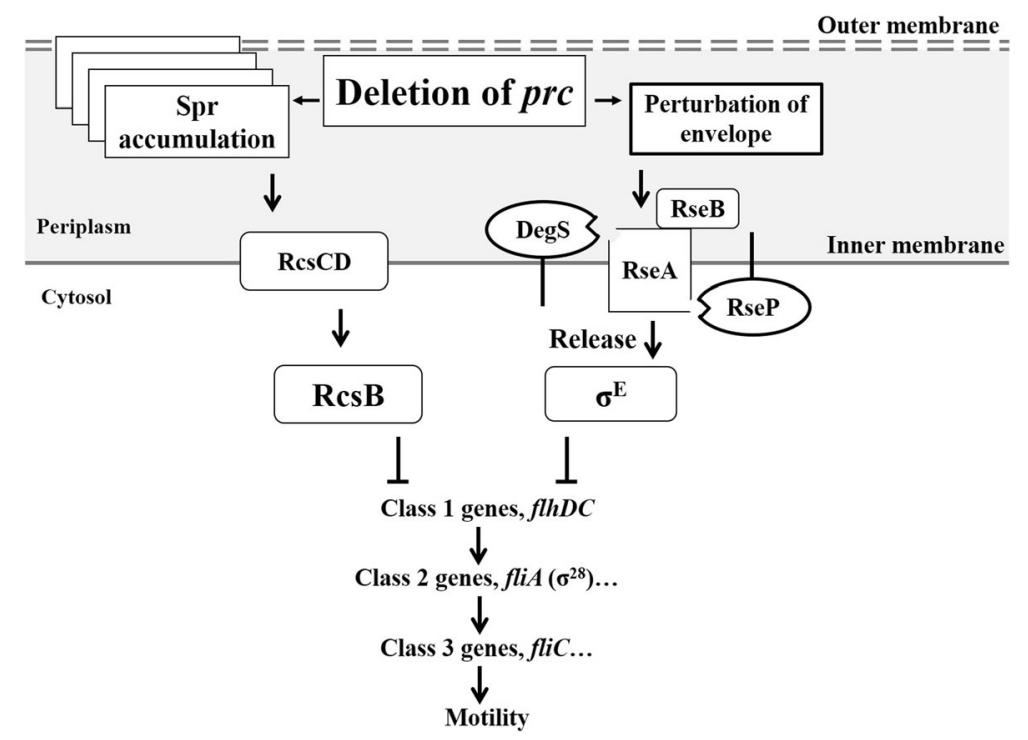

Fig. 14 The model describing how deficiency in the Prc protease leads to defective bacterial motility in E. coli. Prc protease deficiency interferes with proteolytic regulation of its substrates in the bacterial envelope, leading to uncontrolled levels of Prc substrates, such as Spr. The accumulation of the substrates may alter the OMP profile, leading to compromised OM integrity and disturbing peptidoglycan biogenesis. The resulting disturbance in the envelope triggers activation of the $\sigma^{E}$ or RcsCDB extracytoplasmic stress response systems. The activated $\sigma^{E}$ and RcsCDB systems decrease flagellar biosynthesis and thus bacterial motility

\section{Conclusions}

In addition to our previous finding that lacking of Prc resulted in decreased ability of ExPEC to cause bacteremia [4], we demonstrated herein that such defect can also diminish the ability of ExPEC to cause UTIs. Given prc contributes to ExPEC infections in different extraintestinal host tissues as found in the bloodstream and UT, our study strengthens an idea that Prc or Prc homologs may be a potential antimicrobial target for developing a novel strategy in managing ExPEC or other bacterial infections. In addition, the $\sigma^{\mathrm{E}}$ and RcsCDB systems, which are responsible for the defect of the prc mutant in causing UTIs, are potential antimicrobial targets in the same light.

\section{Supplementary information}

Supplementary information accompanies this paper at https://doi.org/10. 1186/s12929-019-0605-y.

Additional file 1: Table S1. Primers used in this study

Additional file 2: Table S2. Identification of altered total proteins by liquid chromatography-tandem mass spectrometry

Additional file 3: Table S3. The differentially expressed genes in $\Delta p r c-$ RS218 compared to those in WT-RS218 by RNA-seq

Additional file 4: Figure S1. The effects of overexpression of DegQ on motility, FliC expression, and activation of $\sigma^{E}$ signaling. (a) Motility diameter of WT-RS218/pACYC184, $\Delta$ prc-RS218/pACYC184, and $\Delta$ prcRS218/pDegQ. (b) Flic levels in WT-RS218/pACYC184, $\Delta$ prc-RS218/ pACYC184, and $\Delta$ prc-RS218/pDegQ. (c) Promoter activity of degP in WTRS218/pACYC184, $\Delta$ prc-RS218/pACYC184, and $\Delta$ prc-RS218/pDegQ. degP is positively regulated by the $\sigma^{E}$ signaling system. The promoter activity of degP can reflect the activation level of $\sigma^{E}$ signaling

\section{Abbreviations}

2CSTSs: Two-component signal transduction systems; CFU: Colony-Forming unit; co-RNAP: core RNA polymerase; ESSSs: Extracytoplasmic stress signaling systems; EtBr: Ethidium bromide; ExPEC: Extraintestinal pathogenic E. coli; FPKM: Fragments per kilobase of transcript per million mapped reads; holoRNAP: RNA polymerase holoenzyme; hpi: Hour post-inoculation; IACUC: Institutional Animal Care and Use Committee; IM: Inner membrane; LB: Luria Bertani; LC/MS/MS: Liquid chromatography-tandem mass spectrometry; M-MLV: Moloney murine leukemia virus; OM: Outer membrane; OMPs: OM-associated proteins; PCR: Polymerase chain reaction; qPCR: Realtime quantitative PCR; RNA-seq: RNA sequencing; RT-PCR: Reverse transcription-PCR; UT: Urinary tract; UTIs: Urinary tract infections

\section{Acknowledgments}

We thank Dr. Shainn-Wei Wang for helpful discussions.

\section{Authors' contributions}

WCH carried out the experiments in this study. CYL participated in the transcriptome data analysis and discussion. WCH, MH, and $\mathrm{CHT}$ contributed to the study conception, planning experiments, data analysis and interpretation. JJW and CSC participated in the result discussion and technical support. MCW and WHL contributed materials and technical support. WCH and CHT wrote the manuscript. All authors read and approved the final manuscript

\section{Funding}

This study was supported by grants NSC 102-2628-B-006-004-MY3, MOST 105-2320-B006-023 and MOST 107-2320-B006-024 from the Ministry of Science and Technology, Taiwan. The funders had no role in study design, data collection and analysis, decision to publish, or preparation of the manuscript.

\section{Availability of data and materials}

All data and materials are fully available without restriction and are within the manuscript and its Additional files.

\section{Ethics approval and consent to participate}

All animal studies were carried out according to the guideline by Council of Agriculture Executive Yuan Guideline for the Care and Use of Laboratory 
Animals, Republic of China. All of the animal experimental procedures were reviewed and approved by the Institutional Animal Care and Use Committee (IACUC) of National Cheng Kung University, Tainan City, Taiwan (Approval number. 102101 and 105175).

\section{Consent for publication}

Not applicable.

\section{Competing interests}

The authors declare that they have no competing interests.

\section{Author details}

${ }^{1}$ Institute of Molecular Medicine, College of Medicine, National Cheng Kung University, Tainan, Taiwan. ${ }^{2}$ Institute of Basic Medical Sciences, College of Medicine, National Cheng Kung University, Tainan, Taiwan. ${ }^{3}$ Institute of Information Science, Academia Sinica, Taipei, Taiwan. ${ }^{4}$ Center of Infectious Disease and Signaling Research, National Cheng Kung University, Tainan, Taiwan. ${ }^{5}$ Department of Biotechnology and Laboratory Science in Medicine, School of Biomedical Science and Engineering, National Yang Ming University, Taipei, Taiwan. ${ }^{6}$ Department of Internal Medicine, National Cheng Kung University Hospital, College of Medicine, National Cheng Kung University, Tainan, Taiwan. ${ }^{7}$ Department of Biochemistry and Molecular Biology, College of Medicine, National Cheng Kung University, Tainan, Taiwan.

\section{Received: 22 August 2019 Accepted: 19 December 2019} Published online: 03 January 2020

\section{References}

1. Poolman JT, Wacker M. Extraintestinal pathogenic Escherichia coli, a common human pathogen: challenges for vaccine development and progress in the field. J Infect Dis. 2016;213(1):6-13.

2. Mellata M. Human and avian extraintestinal pathogenic Escherichia coli: infections, zoonotic risks, and antibiotic resistance trends. Foodborne Pathog Dis. 2013;10(11):916-32

3. Vasoo S, Barreto JN, Tosh PK. Emerging issues in gram-negative bacterial resistance: an update for the practicing clinician. Mayo Clin Proc. 2015;90(3):395-403.

4. Wang CY, Wang SW, Huang WC, Kim KS, Chang NS, Wang YH, Wu MH, Teng CH. Prc contributes to Escherichia coli evasion of classical complement-mediated serum killing. Infect Immun. 2012;80(10):3399-409.

5. Baumler AJ, Kusters JG, Stojiljkovic I, Heffron F. Salmonella typhimurium loci involved in survival within macrophages. Infect Immun. 1994;62(5):1623-30.

6. Bandara AB, Sriranganathan N, Schurig GG, Boyle SM. Carboxyl-terminal protease regulates Brucella suis morphology in culture and persistence in macrophages and mice. J Bacteriol. 2005;187(16):5767-75.

7. Bandara AB, DeShazer D, Inzana TJ, Sriranganathan N, Schurig GG, Boyle SM. A disruption of ctpA encoding carboxy-terminal protease attenuates Burkholderia mallei and induces partial protection in CD1 mice. Microb Pathog. 2008;45(3):207-16.

8. Deng CY, Deng AH, Sun ST, Wang L, Wu J, Wu Y, Chen XY, Fang RX, Wen TY, Qian W. The periplasmic PDZ domain-containing protein Prc modulates full virulence, envelops stress responses, and directly interacts with dipeptidyl peptidase of Xanthomonas oryzae pv. Oryzae. Mol Plant-Microbe Interact. 2014;27(2):101-12.

9. Liao CT, Liu YF, Chiang YC, Lo HH, Du SC, Hsu PC, Hsiao YM. Functional characterization and transcriptome analysis reveal multiple roles for prc in the pathogenicity of the black rot pathogen Xanthomonas campestris pv. Campestris. Res Microbiol. 2016;167(4):299-312.

10. Godlewska R, Wisniewska K, Pietras Z, Jagusztyn-Krynicka EK. Peptidoglycanassociated lipoprotein (pal) of gram-negative bacteria: function, structure, role in pathogenesis and potential application in immunoprophylaxis. FEMS Microbiol Lett. 2009;298(1):1-11.

11. Duan Q, Zhou M, Zhu L, Zhu G. Flagella and bacterial pathogenicity. J Basic Microbiol. 2013.

12. Lane MC, Lockatell V, Monterosso G, Lamphier D, Weinert J, Hebel JR, Johnson $\mathrm{DE}$, Mobley HL. Role of motility in the colonization of uropathogenic Escherichia coli in the urinary tract. Infect Immun. 2005;73(11):7644-56.

13. Wright KJ, Seed PC, Hultgren SJ. Uropathogenic Escherichia coli flagella aid in efficient urinary tract colonization. Infect Immun. 2005;73(11):7657-68.
14. Lane MC, Alteri CJ, Smith SN, Mobley HL. Expression of flagella is coincident with uropathogenic Escherichia coli Ascension to the upper urinary tract. Proc Natl Acad Sci U S A. 2007;104(42):16669-74.

15. Schwan WR. Flagella allow uropathogenic Escherichia coli Ascension into murine kidneys. Int J Med Microbiol : IJMM. 2008;298(5-6):441-7.

16. Terashima H, Kojima S, Homma M. Flagellar motility in bacteria structure and function of flagellar motor. Int Rev Cell Mol Biol. 2008;270:39-85.

17. Wang S, Fleming RT, Westbrook EM, Matsumura P, McKay DB. Structure of the Escherichia coli FlhDC complex, a prokaryotic heteromeric regulator of transcription. J Mol Biol. 2006;355(4):798-808.

18. Soutourina OA, Bertin PN. Regulation cascade of flagellar expression in gram-negative bacteria. FEMS Microbiol Rev. 2003;27(4):505-23.

19. Shin S, Park C. Modulation of flagellar expression in Escherichia coli by acetyl phosphate and the osmoregulator OmpR. J Bacteriol. 1995;177(16):4696-702.

20. De Wulf $\mathrm{P}$, Kwon O, Lin EC. The CpxRA signal transduction system of Escherichia coli: growth-related autoactivation and control of unanticipated target operons. J Bacteriol. 1999;181(21):6772-8.

21. Francez-Charlot A, Laugel B, Van Gemert A, Dubarry N, Wiorowski F, CastanieCornet MP, Gutierrez C, Cam K. RcsCDB his-asp phosphorelay system negatively regulates the fhDC operon in Escherichia coli. Mol Microbiol. 2003;49(3):823-32.

22. Kostakioti M, Hadjifrangiskou M, Pinkner JS, Hultgren SJ. QseC-mediated dephosphorylation of QseB is required for expression of genes associated with virulence in uropathogenic Escherichia coli. Mol Microbiol. 2009;73(6):1020-31.

23. Price NL, Raivio TL. Characterization of the Cpx regulon in Escherichia coli strain MC4100. J Bacteriol. 2009;191(6):1798-815.

24. Evans KL, Kannan S, Li G, de Pedro MA, Young KD. Eliminating a set of four penicillin binding proteins triggers the Rcs phosphorelay and Cpx stress responses in Escherichia coli. J Bacteriol. 2013;195(19):4415-24.

25. Raina S, Missiakas D, Georgopoulos $C$. The rpoE gene encoding the sigma $E$ (sigma 24) heat shock sigma factor of Escherichia coli. EMBO J. 1995;14(5):1043-55.

26. Rouviere PE, De Las Penas A, Mecsas J, Lu CZ, Rudd KE, Gross CA. rpoE, the gene encoding the second heat-shock sigma factor, sigma $\mathrm{E}$, in Escherichia coli. EMBO J. 1995;14(5):1032-42.

27. Shi W, Li C, Louise CJ, Adler J. Mechanism of adverse conditions causing lack of flagella in Escherichia coli. J Bacteriol. 1993;175(8):2236-40.

28. Ades SE, Connolly LE, Alba BM, Gross CA. The Escherichia coli sigma(E)dependent extracytoplasmic stress response is controlled by the regulated proteolysis of an anti-sigma factor. Genes Dev. 1999;13(18):2449-61.

29. Tam C, Collinet B, Lau G, Raina S, Missiakas D. Interaction of the conserved region 4.2 of sigma(E) with the RseA anti-sigma factor. J Biol Chem. 2002; 277(30):27282-7.

30. Campbell EA, Tupy JL, Gruber TM, Wang S, Sharp MM, Gross CA, Darst SA. Crystal structure of Escherichia coli sigmaE with the cytoplasmic domain of its anti-sigma RseA. Mol Cell. 2003;11(4):1067-78.

31. Alba BM, Leeds JA, Onufryk C, Lu CZ, Gross CA. DegS and YaeL participate sequentially in the cleavage of RseA to activate the sigma(E)-dependent extracytoplasmic stress response. Genes Dev. 2002;16(16):2156-68.

32. Kanehara K, Ito K, Akiyama Y. YaeL (EcfE) activates the sigma(E) pathway of stress response through a site-2 cleavage of anti-sigma(E). RseA Genes Dev. 2002;16(16):2147-55

33. Akiyama Y, Kanehara K, Ito K. RseP (YaeL), an Escherichia coli RIP protease, cleaves transmembrane sequences. EMBO J. 2004;23(22):4434-42.

34. Flynn JM, Levchenko I, Sauer RT, Baker TA. Modulating substrate choice: the $\mathrm{SspB}$ adaptor delivers a regulator of the extracytoplasmic-stress response to the AAA+ protease ClpXP for degradation. Genes Dev. 2004;18(18):2292-301.

35. Alba BM, Zhong HJ, Pelayo JC, Gross CA. degS (hhoB) is an essential Escherichia coli gene whose indispensable function is to provide sigma (E) activity. Mol Microbiol. 2001;40(6):1323-33.

36. Walsh NP, Alba BM, Bose B, Gross CA, Sauer RT. OMP peptide signals initiate the envelope-stress response by activating DegS protease via relief of inhibition mediated by its PDZ domain. Cell. 2003;113(1):61-71.

37. Silhavy TJ, Kahne D, Walker S. The bacterial cell envelope. Cold Spring Harb Perspect Biol. 2010;2(5):a000414.

38. Singh SK, Parveen S, SaiSree L, Reddy M. Regulated proteolysis of a crosslink-specific peptidoglycan hydrolase contributes to bacterial morphogenesis. Proc Natl Acad Sci U S A. 2015;112(35):10956-61.

39. Kao JS, Stucker DM, Warren JW, Mobley HL. Pathogenicity island sequences of pyelonephritogenic Escherichia coli CFT073 are associated with virulent uropathogenic strains. Infect Immun. 1997;65(7):2812-20.

40. Chen SL, Hung CS, Xu J, Reigstad CS, Magrini V, Sabo A, Blasiar D, Bieri T, Meyer RR, Ozersky P, Armstrong JR, Fulton RS, Latreille JP, Spieth J, Hooton 
TM, Mardis ER, Hultgren SJ, Gordon Jl. Identification of genes subject to positive selection in uropathogenic strains of Escherichia coli: a comparative genomics approach. Proc Natl Acad Sci U S A. 2006;103(15):5977-82.

41. Lerner CG, Inouye M. Low copy number plasmids for regulated low-level expression of cloned genes in Escherichia coli with blue/white insert screening capability. Nucleic Acids Res. 1990;18(15):4631.

42. Datsenko KA, Wanner BL. One-step inactivation of chromosomal genes in Escherichia coli K-12 using PCR products. Proc Natl Acad Sci U S A. 2000;97(12):6640-5.

43. Yamamoto H, Kurosawa S, Sekiguchi J. Localization of the vegetative cell wall hydrolases LytC, LytE, and LytF on the Bacillus subtilis cell surface and stability of these enzymes to cell wall-bound or extracellular proteases. J Bacteriol. 2003; 185(22):6666-77.

44. Teng CH, Tseng YT, Maruvada R, Pearce D, Xie Y, Paul-Satyaseela M, Kim KS. $\mathrm{Nlpl}$ contributes to Escherichia coli K1 strain RS218 interaction with human brain microvascular endothelial cells. Infect Immun. 2010;78(7):3090-6.

45. Wijetunge DS, Katani R, Kapur V, Kariyawasam S. Complete genome sequence of Escherichia coli strain RS218 (O18:H7:K1), associated with neonatal meningitis. Genome Announc. 2015;3(4).

46. Mortazavi A, Williams BA, McCue K, Schaeffer L, Wold B. Mapping and quantifying mammalian transcriptomes by RNA-Seq. Nat Methods. 2008;5(7):621-8.

47. Gama-Castro S, Salgado H, Santos-Zavaleta A, Ledezma-Tejeida D, MunizRascado L, Garcia-Sotelo JS, Alquicira-Hernandez K, Martinez-Flores I, Pannier L, Castro-Mondragon JA, Medina-Rivera A, Solano-Lira H, Bonavides-Martinez C, Perez-Rueda E, Alquicira-Hernandez S, Porron-Sotelo L, Lopez-Fuentes A, Hernandez-Koutoucheva A, Del Moral-Chavez V, Rinaldi F, Collado-Vides J. RegulonDB version 9.0: high-level integration of gene regulation, coexpression, motif clustering and beyond. Nucleic Acids Res. 2016;44(D1):D133-43.

48. He XL, Wang Q, Peng L, Qu YR, Puthiyakunnon S, Liu XL, Hui CY, Boddu S, Cao H, Huang SH. Role of uropathogenic Escherichia coli outer membrane protein T in pathogenesis of urinary tract infection. Pathog Dis. 2015;73(3).

49. Davalos-Garcia M, Conter A, Toesca I, Gutierrez C, Cam K. Regulation of osmC gene expression by the two-component system rcsB-rcsC in Escherichia coli. J Bacteriol. 2001;183(20):5870-6.

50. De Wulf P, McGuire AM, Liu X, Lin EC. Genome-wide profiling of promoter recognition by the two-component response regulator CpxR-P in Escherichia coli. J Biol Chem. 2002:277(29):26652-61.

51. Rezuchova B, Miticka H, Homerova D, Roberts M, Kormanec J. New members of the Escherichia coli sigmaE regulon identified by a two-plasmid system. FEMS Microbiol Lett. 2003;225(1):1-7.

52. Ferrieres L, Aslam SN, Cooper RM, Clarke DJ. The yjbEFGH locus in Escherichia coli K-12 is an operon encoding proteins involved in exopolysaccharide production. Microbiology. 2007;153(Pt 4):1070-80.

53. Yamamoto $\mathrm{K}$, Ishihama A. Characterization of copper-inducible promoters regulated by CpxA/CpxR in Escherichia coli. Biosci Biotechnol Biochem. 2006;70(7):1688-95.

54. Rhodius VA, Suh WC, Nonaka G, West J, Gross CA. Conserved and variable functions of the sigmaE stress response in related genomes. PLoS Biol. 2006;4(1):e2.

55. Lan CY, Igo MM. Differential expression of the OmpF and OmpC porin proteins in Escherichia coli K-12 depends upon the level of active OmpR. J Bacteriol. 1998:180(1):171-4.

56. Clarke MB, Sperandio V. Transcriptional autoregulation by quorum sensing Escherichia coli regulators B and C (QseBC) in enterohaemorrhagic E. coli (EHEC). Mol Microbiol. 2005;58(2):441-55.

57. Maris $\mathrm{AE}$, Walthers $\mathrm{D}$, Mattison $\mathrm{K}$, Byers $\mathrm{N}$, Kenney $\mathrm{L}$. The response regulator OmpR oligomerizes via beta-sheets to form head-to-head dimers. J Mol Biol. 2005;350(5):843-56.

58. Clausen T, Kaiser M, Huber R, Ehrmann M. HTRA proteases: regulated proteolysis in protein quality control. Nature reviews. Molecular cell biology. 2011;12(3):152-62.

59. Brill JA, Quinlan-Walshe C, Gottesman S. Fine-structure mapping and identification of two regulators of capsule synthesis in Escherichia coli K-12. J Bacteriol. 1988;170(6):2599-611.

60. Keiler KC, Sauer RT. Identification of active site residues of the Tsp protease. J Biol Chem. 1995;270(48):28864-8.

61. Cho SH, Szewczyk J, Pesavento C, Zietek M, Banzhaf M, Roszzzenko P, Asmar A, Laloux G, Hov AK, Leverrier P, Van der Henst C, Vertommen D, Typas A, Collet JF. Detecting envelope stress by monitoring beta-barrel assembly. Cell. 2014;159(7):1652-64.

62. Farris C, Sanowar S, Bader MW, Pfuetzner R, Miller SI. Antimicrobial peptides activate the Rcs regulon through the outer membrane lipoprotein RcsF. J Bacteriol. 2010;192(19):4894-903.

63. Laubacher ME, Ades SE. The Rcs phosphorelay is a cell envelope stress response activated by peptidoglycan stress and contributes to intrinsic antibiotic resistance. J Bacteriol. 2008;190(6):2065-74.
64. Majdalani N, Gottesman S. The Rcs phosphorelay: a complex signal transduction system. Annu Rev Microbiol. 2005;59:379-405.

65. Singh SK, SaiSree L, Amrutha RN, Reddy M. Three redundant murein endopeptidases catalyse an essential cleavage step in peptidoglycan synthesis of Escherichia coli K12. Mol Microbiol. 2012;86(5):1036-51.

66. Schwechheimer C, Rodriguez DL, Kuehn MJ. Nlpl-mediated modulation of outer membrane vesicle production through peptidoglycan dynamics in Escherichia coli. MicrobiologyOpen. 2015;4(3):375-89.

67. Aramini JM, Rossi P, Huang YJ, Zhao L, Jiang M, Maglaqui M, Xiao R, Locke J, Nair R, Rost B, Acton TB, Inouye M, Montelione GT. Solution NMR structure of the $\mathrm{NlpC/P60} \mathrm{domain} \mathrm{of} \mathrm{lipoprotein} \mathrm{Spr} \mathrm{from} \mathrm{Escherichia} \mathrm{coli:} \mathrm{structural} \mathrm{evidence} \mathrm{for}$ a novel cysteine peptidase catalytic triad. Biochem. 2008;47(37):9715-7.

68. Lima S, Guo MS, Chaba R, Gross CA, Sauer RT. Dual molecular signals mediate the bacterial response to outer-membrane stress. Sci. 2013; 340(6134):837-41.

69. Malet H, Canellas F, Sawa J, Yan J, Thalassinos K, Ehrmann M, Clausen T, Saibil HR. Newly folded substrates inside the molecular cage of the HtrA chaperone DegQ. Nat Struct Mol Biol. 2012;19(2):152-7.

70. Chimalakonda G, Ruiz N, Chng SS, Garner RA, Kahne D, Silhavy TJ. Lipoprotein LptE is required for the assembly of LptD by the beta-barre assembly machine in the outer membrane of Escherichia coli. Proc Natl Acad Sci U S A. 2011:108(6):2492-7.

71. Noinaj N., Rollauer S.E. and Buchanan S.K. The beta-barrel membrane protein insertase machinery from Gram-negative bacteria. Curr Opin Struct Biol. 2015;31(35-42.

72. Blaszczak A, Zylicz M, Georgopoulos C, Liberek K. Both ambient temperature and the DnaK chaperone machine modulate the heat shock response in Escherichia coli by regulating the switch between sigma 70 and sigma 32 factors assembled with RNA polymerase. EMBO J. 1995;14(20):5085-93.

73. Fahrner KA, Berg HC. Mutations that stimulate flhDC expression in Escherichia coli K-12. J Bacteriol. 2015;197(19):3087-96.

74. Redford P, Roesch PL, Welch RA. DegS is necessary for virulence and is among extraintestinal Escherichia coli genes induced in murine peritonitis. Infect Immun. 2003;71(6):3088-96.

75. Wiles TJ, Kulesus RR, Mulvey MA. Origins and virulence mechanisms of uropathogenic Escherichia coli. Exp Mol Pathol. 2008;85(1):11-9.

76. Miajlovic H, Cooke NM, Moran GP, Rogers TR, Smith SG. Response of extraintestinal pathogenic Escherichia coli to human serum reveals a protective role for Rcs-regulated exopolysaccharide colanic acid. Infect Immun. 2014;82(1):298-305.

77. Li K, Sacks SH, Sheerin NS. The classical complement pathway plays a critical role in the opsonisation of uropathogenic Escherichia coli. Mol Immunol. 2008;45(4):954-62.

78. Plesa M, Hernalsteens JP, Vandenbussche G, Ruysschaert JM, Cornelis P. The SlyB outer membrane lipoprotein of Burkholderia multivorans contributes to membrane integrity. Res Microbiol. 2006;157(6):582-92.

79. Watts RE, Totsika M, Challinor VL, Mabbett AN, Ulett GC, De Voss JJ, Schembri MA. Contribution of siderophore systems to growth and urinary tract colonization of asymptomatic bacteriuria Escherichia coli. Infect Immun. 2012;80(1):333-44.

80. Raivio TL, Leblanc SK, Price NL. The Escherichia coli Cpx envelope stress response regulates genes of diverse function that impact antibiotic resistance and membrane integrity. J Bacteriol. 2013;195(12):2755-67.

81. Miajlovic H, Smith SG. Bacterial self-defence: how Escherichia coli evades serum killing. FEMS Microbiol Lett. 2014;354(1):1-9.

82. Birch RG, Pemberton JM, Basnayake WV. Stable albicidin resistance in Escherichia coli involves an altered outer-membrane nucleoside uptake system. J Gen Microbiol. 1990;136(1):51-8.

83. Bremer E, Middendorf A, Martinussen J, Valentin-Hansen P. Analysis of the tsx gene, which encodes a nucleoside-specific channel-forming protein (Tsx) in the outer membrane of Escherichia coli. Gene. 1990;96(1):59-65.

84. Hashimi SM, Wall MK, Smith AB, Maxwell A, Birch RG. The phytotoxin albicidin is a novel inhibitor of DNA gyrase. Antimicrob Agents Chemother. 2007:51(1):181-7.

85. Seoane A, Sabbaj A, McMurry LM, Levy SB. Multiple antibiotic susceptibility associated with inactivation of the prc gene. J Bacteriol. 1992;174(23):7844-7.

\section{Publisher's Note}

Springer Nature remains neutral with regard to jurisdictional claims in published maps and institutional affiliations. 